\title{
Combined error estimates in the case of dimension reduction
}

\author{
Samrowski, Tatiana
}

\begin{abstract}
We consider the stationary reaction-diffusion problem in a domain $\Omega 3 \Omega \subset \mathbb{R}^{3}$ having the size along one coordinate direction essentially smaller than along the others. By an energy type argumentation, different simplified models of lower dimension can be deduced and solved numerically. For these models, we derive a guaranteed upper bound of the difference between the exact solution of the original problem and a three-dimensional reconstruction generated by the solution of a dimensionally reduced problem. This estimate of the total error is determined as the sum of discretization and modeling errors, which are both explicit and computable. The corresponding discretization errors are estimated by a posteriori estimates of the functional type. Modeling error majorants are also explicitly evaluated. Hence, a numerical strategy based on the balancing modeling and discretization errors can be derived in order to provide an economical way of getting an approximate solution with an a priori given accuracy. Numerical tests are presented and discussed
\end{abstract}

DOI: https://doi.org/10.1515/cmam-2013-0024

Posted at the Zurich Open Repository and Archive, University of Zurich

ZORA URL: https://doi.org/10.5167/uzh-154571

Journal Article

Published Version

Originally published at:

Samrowski, Tatiana (2014). Combined error estimates in the case of dimension reduction. Computational Methods in Applied Mathematics, 14(1):113-134.

DOI: https://doi.org/10.1515/cmam-2013-0024 


\title{
Combined Error Estimates in the Case of Dimension Reduction
}

\author{
Tatiana Samrowski
}

\begin{abstract}
We consider the stationary reaction-diffusion problem in a domain $\Omega \subset \mathbb{R}^{3}$ having the size along one coordinate direction essentially smaller than along the others. By an energy type argumentation, different simplified models of lower dimension can be deduced and solved numerically. For these models, we derive a guaranteed upper bound of the difference between the exact solution of the original problem and a three-dimensional reconstruction generated by the solution of a dimensionally reduced problem. This estimate of the total error is determined as the sum of discretization and modeling errors, which are both explicit and computable. The corresponding discretization errors are estimated by a posteriori estimates of the functional type. Modeling error majorants are also explicitly evaluated. Hence, a numerical strategy based on the balancing modeling and discretization errors can be derived in order to provide an economical way of getting an approximate solution with an a priori given accuracy. Numerical tests are presented and discussed.
\end{abstract}

2010 Mathematical subject classification: 35J20, 65N15, 65N30.

Keywords: Modeling Error, Dimension Reduction, Thin Domain, A Posteriori Error Estimate.

\section{Introduction}

Model simplification is an essential tool in analysis and simulation of many complex physical processes. The method of dimensional reduction is a typical way of solving boundary value problems associated with domains, where the size along one coordinate direction is much smaller than the others. In this case, the solution strategy consists of two steps. First, the original $n$-dimensional problem must be replaced by a simpler $(n-k)$-dimensional one (for the detailed discussion on the hierarchy of reduced problems see, e.g., [2,3]). Then, the simplified model should be numerically solved. The control that the corresponding approximation error is below some given tolerance level can be obtained by applying guaranteed a posteriori estimates of the functional type (see, e.g. $[6,7,11]$ ).

Historically, the subject of error estimation in dimension reduction models was mainly focused on a priori asymptotic error estimates that evaluate the difference between original and reduced models in terms of small (geometric) parameters. In particular, such type of estimates have been properly investigated for diffusion problems and in elasticity theory (cf. $[1,4,5]$ ). Estimates of the different type that can be used to evaluate modeling errors for problems on domains with a given thickness were derived in [13] for diffusion problems, in [12] for stressed planes and in [9] for stationary reaction-diffusion problems.

Tatiana Samrowski

School of Engineering, Zurich University of Applied Sciences,

Technikumstrasse 9, 8401 Winterthur, Switzerland

E-mail: samo@zhaw.ch. 
In this paper, a guaranteed upper bound of the total error in the case of the approximation of the three-dimensional stationary reaction diffusion problem by the two-dimensional model problem is determined as the sum of approximation and modeling errors. It is computable, since the modeling error, which arises due to the replacement of the original problem with a simplified one, is also explicitly estimated. Estimates of this type for stationary diffusion problems with variable coefficients, which may sharply change values and have a complex behavior in the domain, have already been provided and discussed in [10].

The paper is organized as follows. In Section 2, we specify the original problem, introduce the notation, and consider different dimension reduced models. In Section 3, we present the main theorems, which provide guaranteed and fully computable upper bounds of the total error, as well as the approximation and dimensional reduction error majorants. In Section 4, results of numerical tests demonstrate the reliability and efficiency of the combined modelingdiscretization error majorant.

\section{Statement of the Problem and Notation}

Let $\Omega$ be a simply connected and bounded Lipschitz domain defined by

$$
\Omega:=\left\{x \in \mathbb{R}^{3} \mid\left(x_{1}, x_{2}\right) \in \hat{\Omega}, d_{\ominus}\left(x_{1}, x_{2}\right)<x_{3}<d_{\oplus}\left(x_{1}, x_{2}\right)\right\} \in \mathbb{R}^{3},
$$

where $\hat{\Omega} \subset \mathbb{R}^{2}$ denotes the orthogonal projection of $\Omega$ on the $\left(x_{1}, x_{2}\right)$-plane (see Figure 1 ) and $d_{\ominus}$ and $d_{\oplus}$ are Lipschitz continuous and differentiable functions of $\left(x_{1}, x_{2}\right)$-coordinates. We denote the lower and upper faces of $\Omega$ by

$$
\Gamma_{\ominus}:=\left\{x \in \mathbb{R}^{3} \mid\left(x_{1}, x_{2}\right) \in \hat{\Omega}, x_{3}=d_{\ominus}\left(x_{1}, x_{2}\right)\right\}
$$

and

$$
\Gamma_{\oplus}:=\left\{x \in \mathbb{R}^{3} \mid\left(x_{1}, x_{2}\right) \in \hat{\Omega}, x_{3}=d_{\oplus}\left(x_{1}, x_{2}\right)\right\},
$$

respectively, and the lateral boundaries associated with Robin and Dirichlet boundary conditions by

$$
\Gamma_{R}:=\left\{x \in \mathbb{R}^{3} \mid\left(x_{1}, x_{2}\right) \in \hat{\Gamma}_{R}, d_{\ominus}\left(x_{1}, x_{2}\right)<x_{3}<d_{\oplus}\left(x_{1}, x_{2}\right)\right\}
$$

and

$$
\Gamma_{D}:=\left\{x \in \mathbb{R}^{3} \mid\left(x_{1}, x_{2}\right) \in \hat{\Gamma}_{D}, d_{\ominus}\left(x_{1}, x_{2}\right)<x_{3}<d_{\oplus}\left(x_{1}, x_{2}\right)\right\} .
$$

We consider the stationary reaction-diffusion problem with mixed boundary conditions $($ called Problem $\mathcal{P}$ )

$$
\begin{aligned}
-\operatorname{Div}(\mathbf{A} \nabla u)+\rho^{2} u & =f & & \text { in } \Omega, \\
\kappa u+\mathbf{A} \nabla u \cdot \boldsymbol{\nu}_{R} & =0 & & \text { on } \Gamma_{R}, \\
\mathbf{A} \nabla u \cdot \boldsymbol{\nu}_{\ominus} & =F_{\ominus} & & \text { on } \Gamma_{\ominus}, \\
\mathbf{A} \nabla u \cdot \boldsymbol{\nu}_{\oplus} & =F_{\oplus} & & \text { on } \Gamma_{\oplus}, \\
u & =0 & & \text { on } \Gamma_{D},
\end{aligned}
$$

where

$$
f \in L^{2}(\Omega), \quad F_{\ominus} \in L^{2}\left(\Gamma_{\ominus}\right), \quad F_{\oplus} \in L^{2}\left(\Gamma_{\oplus}\right), \quad \rho^{2}(x) \in L^{\infty}(\Omega),
$$

$\boldsymbol{\nu}_{R}, \boldsymbol{\nu}_{\ominus}$ and $\boldsymbol{\nu}_{\oplus}$ are outward normal vectors at $\Gamma_{R}, \Gamma_{\ominus}$ and $\Gamma_{\oplus}$, respectively. We assume that $\kappa>0$ and $\rho>0$ are some known real functions and the diffusion matrix $\mathbf{A}=\left(a_{i j}\right)_{i, j=1,2,3}$ 


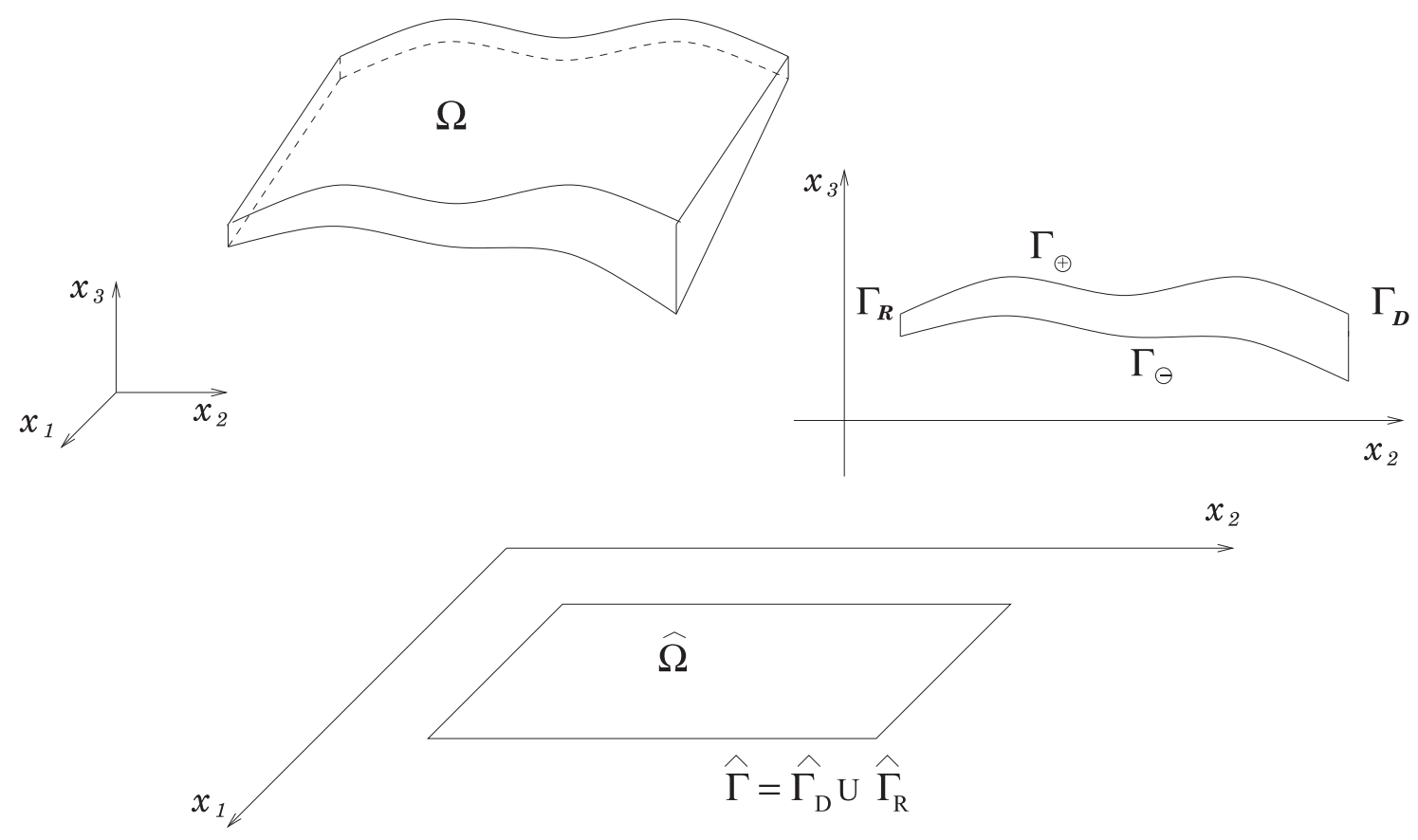

Figure 1. Domain geometry and notation.

has the components from $L_{\infty}(\Omega)$, is symmetric and uniformly positive definite with $0<c_{1}<$ $c_{2}<\infty$ such that

$$
c_{1}|\xi|^{2} \leqslant \mathbf{A} \xi \cdot \xi \leqslant c_{2}|\xi|^{2} \quad \text { for all } \xi \in \mathbb{R}^{3} \quad \text { a.e. in } \Omega \text {. }
$$

We denote by

$$
\mathbf{a}_{i}:=\left\{a_{1 i}, a_{2 i}, a_{3 i}\right\}^{T}, \quad i=1,2,3,
$$

the column vectors of $\mathbf{A}$. The generalized solution

$$
u \in V_{0}:=\left\{v \in H^{1}(\Omega) \mid v=0 \text { on } \Gamma_{D}\right\}
$$

of (1) exists, is unique, continuously depends on the data with respect to the norm of $H^{1}(\Omega)$ and satisfies the following integral relation for all $w \in V_{0}$ :

$$
\int_{\Omega}\left(\mathbf{A} \nabla u \cdot \nabla w+\rho^{2} u w\right) d x+\int_{\Gamma_{R}} \kappa u w d s=\int_{\Omega} f w d x+\int_{\Gamma_{\ominus}} F_{\ominus} w d s+\int_{\Gamma_{\oplus}} F_{\oplus} w d s .
$$

In addition, let

$$
\operatorname{diam} \hat{\Omega} \gg \max _{\left(x_{1}, x_{2}\right) \in \hat{\Omega}} d\left(x_{1}, x_{2}\right)
$$

where $d\left(x_{1}, x_{2}\right)=d_{\oplus}\left(x_{1}, x_{2}\right)-d_{\ominus}\left(x_{1}, x_{2}\right)$ is the domain thickness in point $\left(x_{1}, x_{2}\right)$ and $d\left(x_{1}, x_{2}\right) \geqslant d_{*}>0$ for all $\left(x_{1}, x_{2}\right) \in \overline{\hat{\Omega}}$.

We set

$$
\mathbf{A}_{p}(x):=\left(a_{i j}\right)_{i, j=1,2}: \Omega \rightarrow \mathbb{R}_{\mathrm{sym}}^{2 \times 2}
$$

use the notation

$$
\hat{x}:=\left(x_{1}, x_{2}\right), \quad \hat{x} \in \hat{\Omega},
$$


and mark all functions depending only on $\left(x_{1}, x_{2}\right)$ with ^. With this notation, we define

$$
\hat{F}_{\ominus}:=F_{\ominus}\left(\hat{x}, d_{\ominus}(\hat{x})\right), \quad \hat{F}_{\oplus}:=F_{\oplus}\left(\hat{x}, d_{\oplus}(\hat{x})\right) \quad \text { for any } \hat{x} \in \hat{\Omega},
$$

and note that

$$
\begin{aligned}
\int_{\Gamma_{\ominus}} F_{\ominus} \hat{w} & =\int_{\hat{\Omega}} \hat{F}_{\ominus}(\hat{x}) \hat{w}(\hat{x}) \sqrt{1+\left|\nabla d_{\ominus}(\hat{x})\right|^{2}} \\
\int_{\Gamma_{\oplus}} F_{\oplus} \hat{w} & =\int_{\hat{\Omega}} \hat{F}_{\oplus}(\hat{x}) \hat{w}(\hat{x}) \sqrt{1+\left|\nabla d_{\oplus}(\hat{x})\right|^{2}}
\end{aligned}
$$

Let the operation denote the averaging with respect to $x_{3}$, e.g., for all $g \in L_{1}(\Omega)$

$$
\tilde{g}_{k}(\hat{x}):=\frac{1}{d(\hat{x})} \int_{d_{\ominus}(\hat{x})}^{d_{\oplus}(\hat{x})} x_{3}^{k} g\left(\hat{x}, x_{3}\right) d x_{3} \quad \text { for a.e. } \hat{x} \in \hat{\Omega} .
$$

Further, we set

$$
\mathbf{A}_{p}(x):=\left(a_{i j}(x)\right)_{i, j=1,2}: \Omega \rightarrow \mathbb{R}_{\mathrm{sym}}^{2 \times 2}
$$

and define for $k=0,1,2$

$$
\tilde{\mathbf{A}}_{p, k}(\hat{x}):=\frac{1}{d(\hat{x})} \int_{d_{\ominus}(\hat{x})}^{d_{\oplus}(\hat{x})} x_{3}^{k} \mathbf{A}_{p}(x) d x_{3}
$$

with

$$
\tilde{\mathbf{A}}_{p, k}(\hat{x})=\left(\tilde{a}_{i j}(\hat{x})\right)_{i, j=1,2}: \hat{\Omega} \rightarrow \mathbb{R}_{\mathrm{sym}}^{2 \times 2} .
$$

In what follows, we will distinguish between the three- and two-dimensional divergence operator,

$$
\operatorname{Div} \boldsymbol{\tau}=\frac{\partial \tau_{1}}{\partial x_{1}}+\frac{\partial \tau_{2}}{\partial x_{2}}+\frac{\partial \tau_{3}}{\partial x_{3}}, \quad \operatorname{div} \hat{\boldsymbol{\tau}}=\frac{\partial \hat{\tau}_{1}}{\partial x_{1}}+\frac{\partial \hat{\tau}_{2}}{\partial x_{2}}
$$

and the three- and two-dimensional gradient,

$$
\nabla u:=\left(\frac{\partial u}{\partial x_{1}}, \frac{\partial u}{\partial x_{2}}, \frac{\partial u}{\partial x_{3}}\right), \quad \hat{\nabla} \hat{u}:=\left(\frac{\partial \hat{u}}{\partial x_{1}}, \frac{\partial \hat{u}}{\partial x_{2}}\right) .
$$

We also define

$$
\nabla \hat{u}:=\left(\frac{\partial \hat{u}}{\partial x_{1}}, \frac{\partial \hat{u}}{\partial x_{2}}, 0\right)
$$

and write $\nabla(u-\hat{u})$ for $\nabla u-\nabla \hat{u}$.

Now, we will consider different models of the dimension reduction:

Model 1. In the so-called zero-order reduced model, we assume that the original problem is simplified by a two-dimensional approximate model, which is based on the hypothesis that the exact solution is constant with respect to the $x_{3}$-coordinate. According to this hypothesis, the exact solution $u$ may be well-approximated by a function from the subspace

$$
\begin{gathered}
V_{0}^{(0)}:=\left\{v^{(0)} \in V_{0} \mid \text { there exists } \hat{v}^{(0)} \in H^{1}(\hat{\Omega}) \text { such that } \hat{v}^{(0)}=0 \text { on } \hat{\Gamma}_{D}\right. \text { and } \\
\left.\qquad v^{(0)}(x)=\hat{v}^{(0)}(\hat{x}) \text { for a.e. } x=\left(\hat{x}, x_{3}\right) \in \Omega\right\}
\end{gathered}
$$


(cf. [9]), which is the generalized solution of the following two-dimensional reaction-diffusion problem

$$
\begin{aligned}
-\operatorname{div}\left(d(\hat{x}) \tilde{\mathbf{A}}_{p, 0}(\hat{x}) \hat{\nabla} \hat{u}^{(0)}\right)+d(\hat{x}) \tilde{\rho}_{0}^{2}(\hat{x}) \hat{u}^{(0)} & =d(\hat{x}) \hat{f}_{0}(\hat{x}) & & \text { in } \hat{\Omega}, \\
\tilde{\kappa}_{0}(\hat{x}) \hat{u}^{(0)}+\tilde{\mathbf{A}}_{p, 0}(\hat{x}) \hat{\nabla} \hat{u}^{(0)} \cdot \hat{\boldsymbol{\nu}}_{R} & =0 & & \text { on } \hat{\Gamma}_{R}, \\
\hat{u}^{(0)} & =0 & & \text { on } \hat{\Gamma}_{D},
\end{aligned}
$$

where

$$
\hat{f}(\hat{x}):=\tilde{f}(\hat{x})+\frac{1}{d(\hat{x})}\left(\hat{F}_{\ominus}(\hat{x}) \sqrt{1+\left|\nabla d_{\ominus}(\hat{x})\right|^{2}}+\hat{F}_{\oplus}(\hat{x}) \sqrt{1+\left|\nabla d_{\oplus}(\hat{x})\right|^{2}}\right)
$$

Model 2. For a more sophisticated reduced problem, we accept the hypothesis that the exact solution $u$ is linear with respect to the $x_{3}$-coordinate, and assume that the solution $u$ may be well approximated by a function from the subspace

$$
\begin{gathered}
V_{0}^{(1,0)}:=\left\{v^{(1,0)} \in V_{0} \mid \text { there exists } \hat{v}^{(1,0)} \in H^{1}(\hat{\Omega}) \text { such that } \hat{v}^{(1,0)}=0 \text { on } \hat{\Gamma}_{D}\right. \text { and } \\
\left.\qquad v(x)^{(1,0)}=x_{3} \hat{v}^{(1,0)}(\hat{x}) \text { for a.e. } x=\left(\hat{x}, x_{3}\right) \in \Omega\right\} .
\end{gathered}
$$

For the derivation of the corresponding reduced problem, the functional

$$
\begin{aligned}
J(v):= & \frac{1}{2} \int_{\Omega} \mathbf{A} \nabla v \cdot \nabla v d x+\frac{1}{2} \int_{\Gamma_{R}} \kappa v^{2} d s+\frac{1}{2} \int_{\Omega} \rho^{2} v^{2} d x \\
& -\int_{\Omega} f v d x-\int_{\Gamma_{\ominus}} F_{\ominus} v d s-\int_{\Gamma_{\oplus}} F_{\oplus} v d s
\end{aligned}
$$

should be minimized by the standard arguments of convex analysis. A minimizer

$$
u^{(1,0)}=\underset{v \in V_{0}^{(1,0)}}{\arg \min } J(v)
$$

exists and is unique. Hence, for all $v:=u^{(1,0)}+\alpha w$ with $u^{(1,0)}$ defined by $(5)$, any $w \in V_{0}^{(1,0)}$ and $\alpha \in \mathbb{R}$, the inequality $J(v) \geqslant J\left(u^{(1,0)}\right)$ holds. Due to the definition of the first variation, we have

$$
\partial J\left(u^{(1,0)}, w\right)=\left.\frac{d}{d \alpha} J\left(u^{(1,0)}+\alpha w\right)\right|_{\alpha=0}=T_{1}+T_{2}+T_{3}+T_{4} .
$$

Setting $u^{(1,0)}=x_{3} \hat{u}^{(1,0)}$ and $w=x_{3} \hat{w}$, we obtain

$$
\begin{aligned}
T_{1} & =\left.\frac{1}{\alpha} \int_{\Omega}\left(\frac{1}{2} A \nabla\left(x_{3} \hat{u}^{(1,0)}+\alpha x_{3} \hat{w}\right) \cdot \nabla\left(x_{3} \hat{u}^{(1,0)}+\alpha x_{3} \hat{w}\right)-\frac{1}{2} A \nabla\left(x_{3} \hat{u}^{(1,0)}\right) \cdot \nabla\left(x_{3} \hat{u}^{(1,0)}\right)\right) d x\right|_{\alpha=0} \\
& =\int_{\hat{\Omega}} \tilde{\mathbf{A}}_{p, 2} \hat{\nabla} \hat{u}^{(1,0)} \cdot \hat{\nabla} \hat{w}+\int_{\hat{\Omega}} \tilde{\mathbf{a}}_{3 p, 1} \cdot\left(\hat{\nabla} \hat{u}^{(1,0)} \hat{w}+\hat{\nabla} \hat{w} \hat{u}^{(1,0)}\right)+\int_{\hat{\Omega}} \tilde{a}_{33 p, 0} \hat{u}^{(1,0)} \hat{w} .
\end{aligned}
$$

By similar computations, we find

$$
T_{2}=\int_{\hat{\Omega}} \tilde{\rho}_{p, 2}^{2} \hat{u}^{(1,0)} \hat{w}, \quad T_{3}=\int_{\hat{\Gamma}_{R}} \tilde{\kappa}_{p, 2} \hat{u}^{(1,0)} \hat{w}, \quad \text { and } \quad T_{4}=\int_{\hat{\Omega}} \hat{f}_{p, 1} \hat{w} .
$$

Assuming in addition that

$$
a_{13}=a_{23}=0
$$


we obtain from the minimization problem (6) that the function $u^{(1,0)}$ is the generalized solution of the following two-dimensional reaction-diffusion problem with the homogeneous boundary condition:

$$
\begin{aligned}
-\operatorname{div}\left(d(\hat{x}) \tilde{\mathbf{A}}_{p, 2}(\hat{x}) \hat{\nabla} \hat{u}^{(1,0)}\right)+d(\hat{x})\left(\tilde{\rho}_{2}^{2}(\hat{x})+\frac{1}{d(\hat{x})} \int_{d_{\ominus}}^{d_{\oplus}} a_{33}\right) \hat{u}^{(1,0)} & =d(\hat{x}) \hat{f}_{1}(\hat{x}) & & \text { in } \hat{\Omega}, \\
\tilde{\kappa}_{2}(\hat{x}) \hat{u}^{(1,0)}+\tilde{\mathbf{A}}_{p, 2}(\hat{x}) \hat{\nabla} \hat{u}^{(1,0)} \cdot \hat{\boldsymbol{\nu}}_{R} & =0 & & \text { on } \hat{\Gamma}_{R}, \\
\hat{u}^{(1,0)} & =0 & & \text { on } \hat{\Gamma}_{D} .
\end{aligned}
$$

Model 3. For the next reduced problem (which we hereafter call first-order reduced model), we accept the hypothesis that the exact solution $u$ is affine with respect to the $x_{3}$-coordinate. It was shown in [9] that in the case of (7) the solution $u$ may be well approximated by a function from the subspace

$$
\begin{gathered}
V_{0}^{(1)}:=\left\{v^{(1,1)} \in V_{0} \mid \text { there exists } \hat{v}_{1}^{(1)}, \hat{v}_{2}^{(1)} \in H^{1}(\hat{\Omega}) \text { such that } \hat{v}_{1}^{(1)}=\hat{v}_{2}^{(1)}=0 \text { on } \hat{\Gamma}_{D}\right. \text { and } \\
\left.\qquad v(x)^{(1)}=\hat{v}_{1}^{(1)}(\hat{x})+x_{3} \hat{v}_{2}^{(1)}(\hat{x}) \text { for a.e. } x=\left(\hat{x}, x_{3}\right) \in \Omega\right\},
\end{gathered}
$$

which is the generalized solution of the following two-dimensional reaction-diffusion problem with the homogeneous boundary condition:

$$
\begin{aligned}
& -\operatorname{div} d(\hat{x})\left(\tilde{\mathbf{A}}_{p, 0}(\hat{x}) \hat{\nabla} \hat{u}_{1}^{(1)}+\tilde{\mathbf{A}}_{p, 1}(\hat{x}) \hat{\nabla} \hat{u}_{2}^{(1)}\right) \\
& +d(\hat{x})\left(\tilde{\rho}_{0}^{2}(\hat{x}) \hat{u}_{1}^{(1)}+\tilde{\rho}_{1}^{2}(\hat{x}) \hat{u}_{2}^{(1)}\right)=d(\hat{x}) \hat{f}_{0}(\hat{x}) \quad \text { in } \hat{\Omega}, \\
& -\operatorname{div} d(\hat{x})\left(\tilde{\mathbf{A}}_{p, 1}(\hat{x}) \hat{\nabla} \hat{u}_{1}^{(1)}+\tilde{\mathbf{A}}_{p, 2}(\hat{x}) \hat{\nabla} \hat{u}_{2}^{(1)}\right) \\
& +d(\hat{x})\left(\tilde{\rho}_{1}^{2}(\hat{x}) \hat{u}_{1}^{(1)}+\left(\tilde{\rho}_{2}^{2}(\hat{x})+\frac{1}{d(\hat{x})} \int_{d \ominus}^{d_{\oplus}} a_{33}\right) \hat{u}_{2}^{(1)}\right)=d(\hat{x}) \hat{f}_{1}(\hat{x}) \quad \text { in } \hat{\Omega} \\
& \tilde{\kappa}_{0}(x) \hat{u}_{1}^{(1)}+\tilde{\kappa}_{1}(x) \hat{u}_{2}^{(1)}+\left(\tilde{\mathbf{A}}_{p, 0}(\hat{x}) \hat{\nabla} \hat{u}_{1}^{(1)}+\tilde{\mathbf{A}}_{p, 1}(\hat{x}) \hat{\nabla} \hat{u}_{2}^{(1)}\right) \cdot \hat{\boldsymbol{\nu}}_{R}=0 \quad \text { on } \hat{\Gamma}_{R} \text {, } \\
& \tilde{\kappa}_{1}(x) \hat{u}_{1}^{(1)}+\tilde{\kappa}_{2}(x) \hat{u}_{2}^{(1)}+\left(\tilde{\mathbf{A}}_{p, 1}(\hat{x}) \hat{\nabla} \hat{u}_{1}^{(1)}+\tilde{\mathbf{A}}_{p, 2}(\hat{x}) \hat{\nabla} \hat{u}_{2}^{(1)}\right) \cdot \hat{\boldsymbol{\nu}}_{R}=0 \quad \text { on } \hat{\Gamma}_{R} \text {, } \\
& \hat{u}_{1}^{(1)}=\hat{u}_{2}^{(1)}=0 \quad \text { on } \hat{\Gamma}_{D} \text {. }
\end{aligned}
$$

Remark 2.1. We note that if the domain is bounded by plane parallel faces and the coefficients $a_{i j}(x)$ and $\kappa(x)$ are symmetric functions with respect to $x_{3}$, the system (8) is decomposed into two independent reaction-diffusion problems with the homogeneous boundary conditions

$$
\begin{aligned}
& -\operatorname{div} d(\hat{x}) \tilde{\mathbf{A}}_{p, 0}(\hat{x}) \hat{\nabla} \hat{u}_{1}^{(1)}+d(\hat{x}) \tilde{\rho}_{0}^{2}(\hat{x}) \hat{u}_{1}^{(1)}=d(\hat{x}) \hat{f}_{0}(\hat{x}) \quad \text { in } \hat{\Omega}, \\
& \tilde{\kappa}_{0}(x) \hat{u}_{1}^{(1)}+\tilde{\mathbf{A}}_{p, 0}(\hat{x}) \hat{\nabla} \hat{u}_{1}^{(1)} \cdot \hat{\boldsymbol{\nu}}_{R}=0 \quad \text { on } \hat{\Gamma}_{R}, \\
& \hat{u}_{1}^{(1)}=0 \quad \text { on } \hat{\Gamma}_{D} \text {, } \\
& -\operatorname{div} d(\hat{x}) \tilde{\mathbf{A}}_{p, 2}(\hat{x}) \hat{\nabla} \hat{u}_{2}^{(1)}+d(\hat{x})\left(\tilde{\rho}_{2}^{2}(\hat{x})+\frac{1}{d(\hat{x})} \int_{d_{\ominus}}^{d_{\oplus}} a_{33}\right)(\hat{x}) \hat{u}_{2}^{(1)}=0 \quad \text { in } \hat{\Omega}, \\
& \tilde{\kappa}_{2}(x) \hat{u}_{2}^{(1)}+\tilde{\mathbf{A}}_{p, 2}(\hat{x}) \hat{\nabla} \hat{u}_{2}^{(1)} \cdot \hat{\boldsymbol{\nu}}_{R}=0 \quad \text { on } \hat{\Gamma}_{R}, \\
& \hat{u}_{2}^{(1)}=0 \quad \text { on } \hat{\Gamma}_{D} \text {. }
\end{aligned}
$$

It is obvious that in this case $\hat{u}_{2}^{(1)}=0$, and we obtain the same system as for the zero-order model (cf. (4)). If we wish to improve the zero-order solution, another representation should be used, for example, Model 4. 
Model 4. A second-order model comes from the hypothesis that the exact solution $u$ is quadratic with respect to the $x_{3}$-coordinate. Then, the solution $u$ may be well approximated by a function from the subspace

$$
\begin{gathered}
V_{0}^{(2)}:=\left\{v^{(2)} \in V_{0} \mid \text { there exists } \hat{v}_{1}^{(2)} \in H^{1}(\hat{\Omega}) \text { such that } \hat{v}_{1}^{(2)}=0 \text { on } \hat{\Gamma}_{D}\right. \text { and } \\
\left.\qquad v(x)^{(2)}=x_{3}^{2} \hat{v}_{1}^{(2)}(\hat{x}) \text { for a.e. } x=\left(\hat{x}, x_{3}\right) \in \Omega\right\}
\end{gathered}
$$

and for (7) is the generalized solution of the following two-dimensional reaction-diffusion problem with the homogeneous boundary condition:

$$
\begin{aligned}
-\operatorname{div}\left(d(\hat{x}) \tilde{\mathbf{A}}_{p, 4}(\hat{x}) \hat{\nabla} \hat{u}^{(2)}\right)+d(\hat{x})\left(\tilde{\rho}_{4}^{2}(\hat{x})+\frac{4}{d(\hat{x})} \int_{d_{\ominus}}^{d_{\oplus}} x_{3}^{2} a_{33}\right)(\hat{x}) \hat{u}^{(2)} & =d(\hat{x}) \hat{f}_{2}(\hat{x}) & & \text { in } \hat{\Omega}, \\
\tilde{\kappa}_{4}(\hat{x}) \hat{u}^{(2)}+\tilde{\mathbf{A}}_{p, 4}(\hat{x}) \hat{\nabla} \hat{u}^{(2)} \cdot \hat{\boldsymbol{\nu}}_{R} & =0 & & \text { on } \hat{\Gamma}_{R}, \\
\hat{u}^{(2)} & =0 & & \text { on } \hat{\Gamma}_{D} .
\end{aligned}
$$

\section{Estimation of the Deviation from the Exact Solution}

\subsection{Combined Error Estimate}

Our analysis of the deviation from the exact solution is based upon the so-called functionaltype a posteriori error estimates (see [6-10] and the references cited therein). More precisely, we use such type estimate derived for the reaction-diffusion equation with mixed boundary conditions (see [8, estimates $(4.2 .21),(4.2 .22)]$ and [9]) for the combined error norm

$$
\|u-v\| \|:=\left(\|\nabla(u-v)\|_{A}^{2}+\|\rho(u-v)\|_{L_{2}(\Omega)}^{2}+\left\|\kappa^{1 / 2}(u-v)\right\|_{L_{2}\left(\Gamma_{R}\right)}^{2}\right)^{1 / 2},
$$

where

$$
\|\nabla(u-v)\|_{A}^{2}:=\int_{\Omega} \mathbf{A}(x) \nabla(u-v) \cdot \nabla(u-v) d x .
$$

In the following, we denote the exact solution of the stationary reaction-diffusion problem considered in Section 2 by $u$, the exact solution of the simplified $m$-order model by $u^{(m)}$ and the numerical solution of the simplified $m$-order model by $u_{h}^{(m)}$. A combined modelingdiscretization estimate of the total error is presented by the following theorem:

Theorem 3.1. The total error is bounded from above by the sum

$$
\left\|\nabla\left(u-u_{h}^{(m)}\right)\right\| \leqslant E_{\bmod }^{(m), h}+E_{\mathrm{disc}}^{h},
$$

where $E_{\bmod }^{(m), h}$ and $E_{\text {disc }}^{h}$ are generated by the modeling and the discretization parts of the error, respectively, and are defined and estimated as follows:

$$
\begin{aligned}
E_{\mathrm{disc}}^{h} & :=\left\|\nabla\left(u^{(m)}-u_{h}^{(m)}\right)\right\| \| \\
E_{\bmod }^{(m), h} & :=\left\|\nabla\left(u-u^{(m)}\right)\right\| \| \leqslant M_{\bmod }\left(u_{h}^{(m)}, \mathbf{y}\right)+E_{\mathrm{disc}}^{h}
\end{aligned}
$$

where

$$
\begin{aligned}
M_{\text {mod }}\left(u_{h}^{(m)}, \mathbf{y}\right):= & \sqrt{\breve{M}_{1}^{2}\left(u_{h}^{(m)}, \mathbf{y}\right)+\breve{M}_{2}^{2}\left(u_{h}^{(m)}, \mathbf{y}\right)+\breve{M}_{3 R}\left(u_{h}^{(m)}, \mathbf{y}\right)} \\
& +c_{1}^{-1}\left(C_{\Gamma_{\ominus}} \breve{M}_{3 \ominus}\left(u_{h}^{(m)}, \mathbf{y}\right)+C_{\Gamma_{\oplus}} \breve{M}_{3 \oplus}\left(u_{h}^{(m)}, \mathbf{y}\right)\right)
\end{aligned}
$$


with

$$
\begin{aligned}
\breve{M}_{1}\left(u_{h}^{(m)}, \mathbf{y}\right) & :=\left(\int_{\Omega}\left(\nabla u_{h}^{(m)}-\mathbf{A}^{-1} \mathbf{y}\right) \cdot\left(\mathbf{A} \nabla u_{h}^{(m)}-\mathbf{y}\right) d x\right)^{1 / 2}, \\
\breve{M}_{2}\left(u_{h}^{(m)}, \mathbf{y}\right) & :=\left\|\rho^{-1}\left(\operatorname{Div} \mathbf{y}-\rho^{2} u_{h}^{(m)}+f\right)\right\|_{L_{2}(\Omega)}, \\
\breve{M}_{3 R}\left(u_{h}^{(m)}, \mathbf{y}\right) & :=\left\|\kappa^{-1 / 2}\left(\kappa u_{h}^{(m)}+\mathbf{y} \cdot \boldsymbol{\nu}_{R}\right)\right\|_{L_{2}\left(\Gamma_{R}\right)}, \\
\breve{M}_{3 \ominus}\left(u_{h}^{(m)}, \mathbf{y}\right) & :=\left\|F_{\ominus}-\mathbf{y} \cdot \boldsymbol{\nu}_{\ominus}\right\|_{L_{2}\left(\Gamma_{\ominus}\right)}, \\
\breve{M}_{3 \oplus}\left(u_{h}^{(m)}, \mathbf{y}\right) & :=\left\|F_{\oplus}-\mathbf{y} \cdot \boldsymbol{\nu}_{\ominus}\right\|_{L_{2}\left(\Gamma_{\oplus}\right)}
\end{aligned}
$$

for all

$$
\begin{array}{r}
\mathbf{y} \in H^{+}(\Omega, \operatorname{Div}):=\left\{\mathbf{y} \in L_{2}\left(\Omega, \mathbb{R}^{3}\right) \mid \operatorname{Div} \mathbf{y} \in L_{2}(\Omega), \mathbf{y} \cdot \nu_{\ominus} \in L_{2}\left(\Gamma_{\ominus}\right)\right. \\
\left.\mathbf{y} \cdot \nu_{\oplus} \in L_{2}\left(\Gamma_{\oplus}\right), \mathbf{y} \cdot \nu_{R} \in L_{2}\left(\Gamma_{R}\right)\right\}
\end{array}
$$

Proof. In the case of the approximation of (1) by an $m$-order reduced model, it follows by the triangle inequality for the total deviation of the exact solution that

$$
\left\|u-u_{h}^{(m)} \mid\right\| \leqslant\left\|u-u^{(m)}\right\|\|+\| u^{(m)}-u_{h}^{(m)}\|\|=: E_{\text {mod }}^{(m), h}+E_{\text {disc }}^{h} .
$$

We obtain from $(2)$

$$
\begin{aligned}
& \int_{\Omega}\left(\mathbf{A} \nabla\left(u-u^{(m)}\right) \cdot \nabla w+\rho^{2}\left(u-u^{(m)}\right) w\right) d x+\int_{\Gamma_{R}} \kappa\left(u-u^{(m)}\right) w d s \\
& =\int_{\Omega} f w d x+\int_{\Gamma_{\ominus}} F_{\ominus} w d s+\int_{\Gamma_{\oplus}} F_{\oplus} w d s-\int_{\Omega}\left(\mathbf{A} \nabla u^{(m)} \cdot \nabla w+\rho^{2} u^{(m)} w\right) d x-\int_{\Gamma_{R}} \kappa u^{(m)} w d s
\end{aligned}
$$

for all $w \in V_{0}$ and note that

$$
\int_{\Omega} \operatorname{Div}(\mathbf{y} w) d x=\int_{\Gamma_{\ominus}}\left(\mathbf{y} \cdot \boldsymbol{\nu}_{\ominus}\right) w d s+\int_{\Gamma_{\oplus}}\left(\mathbf{y} \cdot \boldsymbol{\nu}_{\oplus}\right) w d s+\int_{\Gamma_{R}}\left(\mathbf{y} \cdot \nu_{R}\right) w d s .
$$

On the other hand

$$
\int_{\Omega} \operatorname{Div}(\mathbf{y} w) d x=\int_{\Omega}(w \operatorname{Div} \mathbf{y}+\mathbf{y} \cdot \nabla w) d x
$$

Setting $w=u-u^{(m)}$, we find with (9) that

$$
\begin{aligned}
\left(E_{\text {mod }}^{(m), h}\right)^{2}=\int_{\Omega} & \left(\mathbf{y}-\mathbf{A}(x) \nabla\left(u^{(m)}+u_{h}^{(m)}-u_{h}^{(m)}\right)\right) \cdot \nabla\left(u-u^{(m)}\right) d x \\
& +\int_{\Omega}\left(f-\rho^{2}\left(u^{(m)}+u_{h}^{(m)}-u_{h}^{(m)}\right)+\operatorname{Div} \mathbf{y}\right)\left(u-u^{(m)}\right) d x \\
& +\int_{\Gamma_{\ominus}}\left(F_{\ominus}-\mathbf{y} \cdot \boldsymbol{\nu}_{\ominus}\right)\left(u-u^{(m)}\right) d s \\
& +\int_{\Gamma_{\oplus}}\left(F_{\oplus}-\mathbf{y} \cdot \boldsymbol{\nu}_{\oplus}\right)\left(u-u^{(m)}\right) d s \\
& -\int_{\Gamma_{R}}\left(\kappa\left(u^{(m)}+u_{h}^{(m)}-u_{h}^{(m)}\right)+\mathbf{y} \cdot \nu_{R}\right)\left(u-u^{(m)}\right) d s .
\end{aligned}
$$


By Hölder and trace inequalities, we arrive with (14)-(18) at the estimate

$$
\begin{aligned}
\left(E_{\text {mod }}^{(m), h}\right)^{2} \leqslant & \breve{M}_{1}\left(u_{h}^{(m)}, \mathbf{y}\right)\left\|\nabla\left(u-u^{(m)}\right)\right\|_{\mathbf{A}} \\
& +\left\|\nabla\left(u^{(m)}-u_{h}^{(m)}\right)\right\|_{\mathbf{A}}\left\|\nabla\left(u-u^{(m)}\right)\right\|_{\mathbf{A}}+\breve{M}_{2}\left(u_{h}^{(m)}, \mathbf{y}\right)\left\|\rho\left(u-u^{(m)}\right)\right\|_{L^{2}(\Omega)} \\
& +\left\|\rho\left(u^{(m)}-u_{h}^{(m)}\right)\right\|_{L^{2}(\Omega)}\left\|\rho\left(u-u^{(m)}\right)\right\|_{L^{2}(\Omega)}+\breve{M}_{3 R}\left\|\kappa^{1 / 2}\left(u-u^{(m)}\right)\right\|_{L_{2}\left(\Gamma_{R}\right)} \\
& +\left\|\kappa^{1 / 2}\left(u^{(m)}-u_{h}^{(m)}\right)\right\|_{L_{2}\left(\Gamma_{R}\right)}\left\|\kappa^{1 / 2}\left(u-u^{(m)}\right)\right\|_{L_{2}\left(\Gamma_{R}\right)} \\
& +c_{1}^{-1}\left(C_{\Gamma_{\ominus}} \breve{M}_{3 \ominus}\left(u_{h}^{(m)}, \mathbf{y}\right)+C_{\Gamma_{\oplus}} \breve{M}_{3 \oplus}\left(u_{h}^{(m)}, \mathbf{y}\right)\right)\left\|\nabla\left(u-u^{(m)}\right)\right\|_{\mathbf{A}} \\
\leqslant & \left(M_{\bmod }\left(u_{h}^{(m)}, \mathbf{y}\right)+E_{\text {disc }}^{h}\right) E_{\bmod }^{(m), h}
\end{aligned}
$$

where $M_{\bmod }\left(u_{h}^{(m)}, \mathbf{y}\right)$ and $E_{\text {disc }}^{h}$ are defined by (13) and (11), respectively. Division by $E_{\bmod }^{(m), h}$ completes the proof of (12).

Remark 3.1. It is worth noting that the term $M_{\text {mod }}\left(u_{h}^{(m)}, \mathbf{y}\right)$ defined by (13) is very general and can be used for any approximate model with the numerical solution $u_{h}^{(m)}$. Using the specific information of the approximate models, the sharper form of $M_{\bmod }\left(u_{h}^{(m)}, \mathbf{y}\right)$ can be obtained and will be presented in the later section of this paper.

Remark 3.2. If solely a numerical solution with a moderate guaranteed accuracy $\varepsilon$ is required, that means

$$
\left\|u-u_{h}^{(m)}\right\| \mid \leqslant \varepsilon \quad \text { or } \quad \frac{\left\|u-u_{h}^{(m)}\right\|}{\left\|u_{h}^{(m)}\right\|} \leqslant \varepsilon,
$$

one can use Theorem 3.1 for an adaptive modeling-discretization strategy for the solution of stationary reaction-diffusion problems: First, a simplified dimension reduced model should be chosen and numerically solved. Then, the discretization and modeling errors should be controlled with the help of a posteriori estimates. A guaranteed upper bound of the total error is determined as the sum of computable discretization and modeling errors. If

$$
E_{\mathrm{mod}}^{(m), h}+E_{\mathrm{disc}}^{h} \quad \text { or } \quad \frac{E_{\mathrm{mod}}^{(m), h}+E_{\mathrm{disc}}^{h}}{\left\|u_{h}^{(m)}\right\| \|},
$$

respectively, exceeds the given tolerance $\varepsilon$, then either the mesh should be refined (if the discretization error dominates) or a more precise simplified model must be taken (if the modeling error dominates). Hence, the solution process is a combined modeling-discretization strategy for balancing the modeling error $E_{\bmod }^{(m), h}$ and the discretization error $E_{\mathrm{disc}}^{h}$ in a problem-adapted way.

\subsection{A Posteriori Estimation of the Discretization and Modeling Error}

In order to control the numerical discretization error $E_{\text {disc }}^{h}$ defined in (11) for the combined norm (9), we apply the functional-type a posteriori error estimate presented in [9]. For convenience of the reader, here we recall the corresponding theorems, in which the general estimate of the discretization error has been derived (cf. [9, Theorems 4.1]).

Theorem 3.2. (i) For any $u_{h} \in V_{0}$ and any

$$
\begin{array}{r}
\mathbf{y} \in H^{+}(\Omega, \operatorname{Div}):=\left\{\mathbf{y} \in L_{2}\left(\Omega, \mathbb{R}^{3}\right) \mid \operatorname{Div} \mathbf{y} \in L_{2}(\Omega), \mathbf{y} \cdot \nu_{\ominus} \in L_{2}\left(\Gamma_{\ominus}\right),\right. \\
\left.\mathbf{y} \cdot \nu_{\oplus} \in L_{2}\left(\Gamma_{\oplus}\right), \mathbf{y} \cdot \nu_{R} \in L_{2}\left(\Gamma_{R}\right)\right\},
\end{array}
$$


the estimate

$$
\begin{aligned}
\left\|u-u_{h}\right\| \leqslant E_{\mathrm{disc}}^{h}\left(u_{h}, y\right)= & M_{1}\left(u_{h}, \mathbf{y}\right)+C_{F, \Omega} c_{1}^{-1} M_{2}\left(u_{h}, \mathbf{y}\right) \\
& +c_{1}^{-1}\left(C_{\Gamma_{\ominus}} M_{3 \ominus}\left(u_{h}, \mathbf{y}\right)+C_{\Gamma_{\oplus}} M_{3 \oplus}\left(u_{h}, \mathbf{y}\right)+C_{\Gamma_{R}} M_{3 R}\left(u_{h}, \mathbf{y}\right)\right),
\end{aligned}
$$

where

$$
\begin{aligned}
M_{1}\left(u_{h}, \mathbf{y}\right) & :=\left(\int_{\Omega}\left(\nabla u_{h}-\mathbf{A}^{-1} \mathbf{y}\right) \cdot(\mathbf{A} \nabla v-\mathbf{y}) d x\right)^{1 / 2}, \\
M_{2}\left(u_{h}, \mathbf{y}\right) & :=\left\|\operatorname{Div} \mathbf{y}-\rho^{2} u_{h}+f\right\|_{L_{2}(\Omega)}, \\
M_{3 R}\left(u_{h}, \mathbf{y}\right) & :=\left\|\kappa u_{h}+\mathbf{y} \cdot \boldsymbol{\nu}_{R}\right\|_{L_{2}\left(\Gamma_{R}\right)}, \\
M_{3 \ominus}\left(u_{h}, \mathbf{y}\right) & :=\left\|F_{\ominus}-\mathbf{y} \cdot \boldsymbol{\nu}_{\ominus}\right\|_{L_{2}\left(\Gamma_{\ominus}\right)} \\
M_{3 \oplus}\left(u_{h}, \mathbf{y}\right) & :=\left\|F_{\oplus}-\mathbf{y} \cdot \boldsymbol{\nu}_{\ominus}\right\|_{L_{2}\left(\Gamma_{\oplus}\right)}
\end{aligned}
$$

(the constants $C_{F, \Omega}, C_{\Gamma \ominus}, C_{\Gamma \oplus}$ and $C_{\Gamma_{R}}$ come from the corresponding Friedrichs and the trace inequalities), provides an upper bound of the distance to the exact solution $u$ measured in terms of the natural energy norm.

(ii) If $\rho(x)$ and $\kappa(x)$ are strictly positive, then

$$
\begin{aligned}
\left\|u-u_{h}\right\| \leqslant E_{\text {disc }}^{h}\left(u_{h}, y\right)= & \sqrt{M_{1}^{2}\left(u_{h}, \mathbf{y}\right)+\tilde{M}_{2}^{2}\left(u_{h}, \mathbf{y}\right)+\tilde{M}_{3 R}^{2}\left(u_{h}, \mathbf{y}\right)} \\
& +c_{1}^{-1}\left(C_{\Gamma_{\ominus}} M_{3 \ominus}\left(u_{h}, \mathbf{y}\right)+C_{\Gamma_{\oplus}} M_{3 \oplus}\left(u_{h}, \mathbf{y}\right)\right),
\end{aligned}
$$

where

$$
\begin{aligned}
\tilde{M}_{2}\left(u_{h}, \mathbf{y}\right) & :=\left\|\rho^{-1}\left(\operatorname{Div} \mathbf{y}-\rho^{2} u_{h}+f\right)\right\|_{L_{2}(\Omega)}, \\
\tilde{M}_{3 R}\left(u_{h}, \mathbf{y}\right) & :=\left\|\kappa^{-1 / 2}\left(\kappa u_{h}+\mathbf{y} \cdot \boldsymbol{\nu}_{R}\right)\right\|_{L_{2}\left(\Gamma_{R}\right)},
\end{aligned}
$$

suggests another upper bound, which does not involve the constant $C_{F, \Omega}$.

(iii) If, in addition,

$$
\mathbf{y} \in H_{\ominus, \oplus}^{+}(\Omega, \operatorname{Div}):=\left\{\mathbf{y} \in L_{2}\left(\Omega, \mathbb{R}^{3}\right) \mid \operatorname{Div} \mathbf{y} \in L_{2}(\Omega), \mathbf{y} \cdot \nu_{\ominus}=F_{\ominus}, \mathbf{y} \cdot \nu_{\oplus}=F_{\oplus}\right\},
$$

then the distance to $u$ is subject to the estimate

$$
\left\|u-u_{h}\right\|^{2} \leqslant M_{1}^{2}\left(u_{h}, \mathbf{y}\right)+\tilde{M}_{2}^{2}\left(u_{h}, \mathbf{y}\right)+\tilde{M}_{3 R}^{2}\left(u_{h}, \mathbf{y}\right),
$$

which does not contain any constants.

Remark 3.3. We note that Theorem 3.2 can be applied for a two-dimensional problem. In such a case, we have to replace the three-dimensional divergence operator Div by the two-dimensional one.

With (3), (11) and Theorem $3.2(\mathrm{i})$, we conclude for the error related to the numerical treatment of the zero-order model that leads to the solution of the reduced problem (4), which does not possess Neumann boundary conditions:

$$
\begin{aligned}
\left(E_{\text {disc }}^{h}\right)^{2} & =\left\|\hat{u}^{(0)}-\hat{u}_{h}^{(0)}\right\|^{2} \\
& =\int_{\Omega} \mathbf{A} \nabla\left(\hat{u}^{(0)}-\hat{u}_{h}^{(0)}\right) \cdot \nabla\left(\hat{u}^{(0)}-\hat{u}_{h}^{(0)}\right)+\int_{\Omega} \rho^{2}\left(\hat{u}^{(0)}-\hat{u}_{h}^{(0)}\right)^{2}+\int_{\Gamma_{R}} \kappa\left(\hat{u}^{(0)}-\hat{u}_{h}^{(0)}\right)^{2}
\end{aligned}
$$




$$
\begin{aligned}
= & \int_{\hat{\Omega}} d(\hat{x})\left(\tilde{\mathbf{A}}_{p, 0} \nabla\left(\hat{u}^{(0)}-\hat{u}_{h}^{(0)}\right) \cdot \nabla\left(\hat{u}^{(0)}-\hat{u}_{h}^{(0)}\right)+\tilde{\rho}_{0}^{2}\left(\hat{u}^{(0)}-\hat{u}_{h}^{(0)}\right)^{2}\right) \\
& \quad+\int_{\hat{\Gamma}_{R}} d(\hat{x}) \tilde{\kappa}_{0}\left(\hat{u}^{(0)}-\hat{u}_{h}^{(0)}\right)^{2} \\
:= & \hat{M}_{1}\left(\hat{u}_{h}^{(0)}, \hat{\mathbf{y}}\right)+C_{F, \hat{\Omega}_{1}} c_{1}^{-1} \hat{M}_{2}\left(\hat{u}_{h}^{(0)}, \hat{\mathbf{y}}\right)+C_{\hat{\Gamma}_{R}} c_{1}^{-1} \hat{M}_{3 R}\left(\hat{u}_{h}^{(0)}, \hat{\mathbf{y}}\right)
\end{aligned}
$$

with

$$
\begin{aligned}
\hat{M}_{1}\left(\hat{u}_{h}^{(0)}, \hat{\mathbf{y}}\right) & :=\left(\int_{\hat{\Omega}}\left(\nabla \hat{u}_{h}^{(0)}-\left(d(\hat{x}) \tilde{\mathbf{A}}_{p, 0}\right)^{-1} \hat{\mathbf{y}}\right) \cdot\left(d(\hat{x}) \tilde{\mathbf{A}}_{p, 0} \nabla \hat{u}_{h}^{(0)}-\hat{\mathbf{y}}\right) d x\right)^{1 / 2}, \\
\hat{M}_{2}\left(\hat{u}_{h}^{(0)}, \hat{\mathbf{y}}\right) & :=\left\|\operatorname{div} \hat{\mathbf{y}}-d(\hat{x}) \tilde{\rho}_{0}^{2} \hat{u}_{h}^{(0)}+\hat{f}\right\|_{L_{2}(\hat{\Omega})}, \\
\hat{M}_{3 R}\left(\hat{u}_{h}^{(0)}, \hat{\mathbf{y}}\right) & :=\left\|\tilde{\kappa}_{0} \hat{u}_{h}^{(0)}+\hat{\mathbf{y}} \cdot \boldsymbol{\nu}_{R}\right\|_{L_{2}\left(\hat{\Gamma}_{R}\right)}
\end{aligned}
$$

for any $\hat{\mathbf{y}} \in H(\hat{\Omega}, \operatorname{div})$. If $\tilde{\rho}$ and $\tilde{\kappa}$ are strictly positive, then we can obtain the estimate without any constant:

$$
\left\|u-u_{h}\right\|^{2} \leqslant \hat{M}_{1}^{2}\left(\hat{u}_{h}^{(0)}, \hat{\mathbf{y}}\right)+\tilde{M}_{2}^{2}\left(\hat{u}_{h}^{(0)}, \hat{\mathbf{y}}\right)+\tilde{M}_{3 R}^{2}\left(\hat{u}_{h}^{(0)}, \hat{\mathbf{y}}\right),
$$

where

$$
\begin{aligned}
\tilde{M}_{2}\left(\hat{u}_{h}^{(0)}, \hat{\mathbf{y}}\right) & :=\left\|\left(d(\hat{x}) \tilde{\rho}_{0}^{2}\right)^{-1 / 2}\left(\operatorname{div} \hat{\mathbf{y}}-d(\hat{x}) \tilde{\rho}_{0}^{2} \hat{u}_{h}^{(0)}+\hat{f}\right)\right\|_{L_{2}(\hat{\Omega})}, \\
\tilde{M}_{3 R}\left(\hat{u}_{h}^{(0)}, \hat{\mathbf{y}}\right) & :=\left\|\tilde{\kappa}_{0}^{-1 / 2}\left(\tilde{\kappa}_{0} \hat{u}_{h}^{(0)}+\hat{\mathbf{y}} \cdot \boldsymbol{\nu}_{R}\right)\right\|_{L_{2}\left(\hat{\Gamma}_{R}\right)} .
\end{aligned}
$$

All the right-hand side functions are functions of $\hat{x}$. Therefore, we can choose for the reconstruction of the flux a function $\hat{\mathbf{y}} \in H(\hat{\Omega}$, div $)$. For the higher-order models, one can obtain similar estimates for the discretization error, see [9].

The modeling error estimate can also be calculated more exactly dependent on the order of the chosen simplified model. The corresponding statements were proven in [9, Theorem 4.2]:

Theorem 3.3. Let

$$
\mathbf{B}:=\mathbf{A}^{-1}, \quad \mathbf{a}_{3}:=\left\{a_{31}, a_{32}, a_{33}\right\}^{T}, \quad \mathbf{b}_{3}:=\left\{b_{31}, b_{32}, b_{33}\right\}^{T} .
$$

(i) The modeling error of the zero-order reduced model is subject to the following estimate:

$$
\left\|\hat{u}^{(0)}-\hat{u}_{h}^{(0)}\right\| \leqslant M_{1}+M_{2}+M_{3},
$$

where

$$
\begin{aligned}
M_{1}:= & \left(\int_{\hat{\Omega}} d(\hat{x})\left(\tilde{\mathbf{B}}_{p, 0} \tilde{\mathbf{A}}_{p, 0}-\mathbf{I}\right) \hat{\nabla} \hat{u}_{h}^{(0)} \cdot \tilde{\mathbf{A}}_{p, 0} \hat{\nabla} \hat{u}_{h}^{(0)} d \hat{x}\right. \\
& \left.\quad+\int_{\Omega}\left(b_{33} \psi(x)^{2}+2\left(\mathbf{b}_{3} \cdot\left(\tilde{\mathbf{A}}_{p, 0} \hat{\nabla} \hat{u}_{h}^{(0)}, 0\right)\right) \psi(x)\right) d x\right)^{1 / 2}, \\
M_{2}:= & C_{F, \Omega} c_{1}^{-1}\left\|\left(f-\hat{f}_{0}\right)-\left(\rho^{2}-\tilde{\rho}_{0}^{2}\right) \hat{u}_{h}^{(0)}-\frac{\nabla d(\hat{x})}{d(\hat{x})} \cdot \tilde{\mathbf{A}}_{p, 0} \hat{\nabla} \hat{u}_{h}^{(0)}+\frac{\partial \psi}{\partial x_{3}}\right\|_{L_{2}(\Omega)}, \\
M_{3}:= & c_{1}^{-1}\left(C_{\Gamma, R}\left\|\kappa \hat{u}_{h}^{(0)}+\left(\tilde{\mathbf{A}}_{p, 0} \hat{\nabla} \hat{u}_{h}^{(0)}, 0\right) \cdot \boldsymbol{\nu}_{R}\right\|_{L_{2}\left(\Gamma_{R}\right)}\right. \\
& +C_{\Gamma, \ominus}\left\|F_{\ominus}-\left(\tilde{\mathbf{A}}_{p, 0} \hat{\nabla} \hat{u}_{h}^{(0)}, 0\right) \cdot \boldsymbol{\nu}_{\ominus}-\psi \nu_{\ominus 3}\right\|_{L_{2}\left(\Gamma_{\ominus}\right)} \\
& \left.+C_{\Gamma, \oplus}\left\|F_{\oplus}-\left(\tilde{\mathbf{A}}_{p, 0} \hat{\nabla} \hat{u}_{h}^{(0)}, 0\right) \cdot \boldsymbol{\nu}_{\oplus}-\psi \nu_{\oplus 3}\right\|_{L_{2}\left(\Gamma_{\oplus}\right)}\right)
\end{aligned}
$$


with an auxiliary function $\psi$ satisfying the conditions

$$
\psi \in L_{2}(\Omega), \quad \psi \in L_{2}\left(\Gamma_{\ominus}\right), \quad \psi \in L_{2}\left(\Gamma_{\oplus}\right), \quad \psi \in L_{2}\left(\Gamma_{R}\right), \quad \frac{\partial \psi}{\partial x_{3}} \in L_{2}(\Omega) .
$$

(ii) Assume $\rho$ to be strictly positive. Then (20) can be modified to the estimate

$$
\left\|u-\hat{u}_{h}^{(0)}\right\| \mid \leqslant \sqrt{M_{1}^{2}+\tilde{M}_{2}^{2}}+M_{3},
$$

where $M_{1}$ and $M_{3}$ are from (21) and (22), respectively, and the functional $\tilde{M}_{2}^{2}$ is defined by

$$
\tilde{M}_{2}^{2}:=\left\|\rho^{-1}\left(\left(f-\hat{f}_{0}\right)-\left(\rho^{2}-\tilde{\rho}_{0}^{2}\right) \hat{u}_{h}^{(0)}-\frac{\nabla d(\hat{x})}{d(\hat{x})} \cdot \tilde{\mathbf{A}}_{p, 0} \hat{\nabla} \hat{u}_{h}^{(0)}+\frac{\partial \psi}{\partial x_{3}}\right)\right\|_{L_{2}(\Omega)}^{2} .
$$

(iii) If the function $\kappa$ is also strictly positive, then we obtain an upper bound

$$
\left\|u-\hat{u}_{h}^{(0)}\right\| \leqslant \sqrt{M_{1}^{2}+\tilde{M}_{2}^{2}+\tilde{M}_{3 R}^{2}}+M_{3 \ominus \oplus,}
$$

where $M_{1}$ and $\tilde{M}_{2}^{2}$ are from (21) and (24), respectively, and

$$
\begin{aligned}
\tilde{M}_{3 R}:= & \left\|\kappa^{-1 / 2}\left(\kappa \hat{u}_{h}^{(0)}+\left(\tilde{\mathbf{A}}_{p, 0} \hat{\nabla} \hat{u}_{h}^{(0)}, 0\right) \cdot \boldsymbol{\nu}_{R}\right)\right\|_{L_{2}\left(\Gamma_{R}\right)}, \\
M_{3 \oplus \ominus}:= & c_{1}^{-1}\left(C_{\Gamma, \ominus}\left\|F_{\ominus}-\left(\tilde{\mathbf{A}}_{p, 0} \hat{\nabla} \hat{u}_{h}^{(0)}, 0\right) \cdot \boldsymbol{\nu}_{\ominus}-\psi \nu_{\ominus 3}\right\|_{L_{2}\left(\Gamma_{\ominus}\right)}\right. \\
& \left.\quad+C_{\Gamma, \oplus}\left\|F_{\oplus}-\left(\tilde{\mathbf{A}}_{p, 0} \hat{\nabla} \hat{u}_{h}^{(0)}, 0\right) \cdot \boldsymbol{\nu}_{\oplus}-\psi \nu_{\oplus 3}\right\|_{L_{2}\left(\Gamma_{\oplus}\right)}\right) .
\end{aligned}
$$

Remark 3.4. The terms on the right-hand side of (20) have a clear meaning: $M_{1}$ evaluates the error generated by the fact that $\hat{\nabla} \hat{u}_{h}^{(0)}$ does not exactly satisfy the three-dimensional relation, $M_{2}$ represents the error in the equilibrium equation, and $M_{3}$ the error in the boundary condition. We note that for the stationary diffusion problem such an estimate has been earlier derived and tested in [13].

Remark 3.5. The modeling error estimates for Model 2 and Model 4 can be deviated in the same way and have absolutely similar formulation. In the case of Model 2 , the term $\tilde{\mathbf{A}}_{p, 0}$ will be replaced by $\tilde{\mathbf{A}}_{p, 2}$ and for Model 4 by $\tilde{\mathbf{A}}_{p, 4}$. The function $\tilde{\rho}_{0}$ has to be replaced by $\tilde{\rho}_{2}$ (for Model 2 ) and by $\tilde{\rho}_{4}$ (for Model 4 ) and $\hat{f}_{0}$ by $\hat{f}_{1}$ or by $\hat{f}_{2}$, respectively.

Theorem 3.4. (i) The modeling error of the first-order (i.e., $m=1$ in Theorem 3.1) reduced model with the diffusion matrix (7) is subject to the following estimate:

$$
\left\|\hat{u}^{(1)}-\hat{u}_{h}^{(1)}\right\| \leqslant M_{1}+M_{2}+M_{3}
$$

with

$$
\begin{aligned}
M_{1}:= & \int_{\Omega}\left[\hat{u}_{2, h}^{(1)}\left(2 \mathbf{a}_{3} \cdot\left(\hat{\nabla} \hat{u}_{1, h}^{(1)}+x_{3} \hat{\nabla} \hat{u}_{2, h}^{(1)}, 0\right)+a_{33} \hat{u}_{2, h}^{(1)}-\psi\right)+b_{33} \psi^{2}\right. \\
& \left.+2\left(\mathbf{b}_{3} \cdot\left(\tilde{\mathbf{A}}_{p, 0} \hat{\nabla} \hat{u}_{1, h}^{(1)}+\tilde{\mathbf{A}}_{p, 1} \hat{\nabla} \hat{u}_{2, h}^{(1)}, 0\right)\right) \psi\right] d x \\
& +\int_{\hat{\Omega}} d(\hat{x})\left[\tilde{\mathbf{B}}_{p, 0}\left(\left(\tilde{\mathbf{A}}_{p, 0}-\tilde{\mathbf{B}}_{p, 0}^{-1}\right) \hat{\nabla} \hat{u}_{1, h}^{(1)}+\tilde{\mathbf{A}}_{p, 1} \hat{\nabla} \hat{u}_{2, h}^{(1)}\right) \cdot\left(\tilde{\mathbf{A}}_{p, 0} \hat{\nabla} \hat{u}_{1, h}^{(1)}+\tilde{\mathbf{A}}_{p, 1} \hat{\nabla} \hat{u}_{2, h}^{(1)}\right)\right. \\
& \left.\left.+\hat{\nabla} \hat{u}_{2, h}^{(1)} \cdot\left(\tilde{\mathbf{A}}_{p, 1} \hat{\nabla} \hat{u}_{1, h}^{(1)}+\tilde{\mathbf{A}}_{p, 2} \hat{\nabla} \hat{u}_{2, h}^{(1)}\right)\right] d \hat{x}\right)^{1 / 2},
\end{aligned}
$$




$$
\begin{aligned}
M_{2}:= & C_{F, \Omega} c_{1}^{-1} \|\left(f-\hat{f}_{0}\right)-\left(\rho^{2}-\tilde{\rho}_{0}^{2}\right) \hat{u}_{1, h}^{(1)}-\left(x_{3} \rho^{2}-\tilde{\rho}_{1}^{2}-\frac{1}{d(\hat{x})} \int_{d_{\ominus}}^{d_{\oplus}} a_{33}\right) \hat{u}_{2, h}^{(1)} \\
& \quad-\frac{\nabla d(\hat{x})}{d(\hat{x})} \cdot\left(\tilde{\mathbf{A}}_{p, 0} \hat{\nabla} \hat{u}_{1, h}^{(1)}+\tilde{\mathbf{A}}_{p, 1} \hat{\nabla} \hat{u}_{2, h}^{(1)}\right)+\frac{\partial \psi}{\partial x_{3}} \|_{L_{2}(\Omega)}, \\
M_{3}:= & c_{1}^{-1}\left(C_{\Gamma, R}\left\|\kappa\left(\hat{u}_{1, h}^{(1)}+x_{3} \hat{u}_{2, h}^{(1)}\right)+\left(\tilde{\mathbf{A}}_{p, 0} \hat{\nabla} \hat{u}_{1, h}^{(1)}+\tilde{\mathbf{A}}_{p, 1} \hat{\nabla} \hat{u}_{2, h}^{(1)}, 0\right) \cdot \boldsymbol{\nu}_{R}\right\|_{L_{2}\left(\Gamma_{R}\right)}\right. \\
& +C_{\Gamma, \ominus}\left\|F_{\ominus}-\left(\tilde{\mathbf{A}}_{p, 0} \hat{\nabla} \hat{u}_{1, h}^{(1)}+\tilde{\mathbf{A}}_{p, 1} \hat{\nabla} \hat{u}_{2, h}^{(1)}, 0\right) \cdot \boldsymbol{\nu}_{\ominus}-\psi \nu_{\ominus 3}\right\|_{L_{2}\left(\Gamma_{\ominus}\right)} \\
& \left.+C_{\Gamma, \oplus}\left\|F_{\oplus}-\left(\tilde{\mathbf{A}}_{p, 0} \hat{\nabla} \hat{u}_{1, h}^{(1)}+\tilde{\mathbf{A}}_{p, 1} \hat{\nabla} \hat{u}_{2, h}^{(1)}, 0\right) \cdot \boldsymbol{\nu}_{\oplus}-\psi \nu_{\oplus 3}\right\|_{L_{2}\left(\Gamma_{\oplus}\right)}\right),
\end{aligned}
$$

where definitions (3) and (19) are used, and $\psi$ is an auxiliary function satisfying the conditions

$$
\psi \in L_{2}(\Omega), \quad \psi \in L_{2}\left(\Gamma_{R}\right), \quad \psi \in L_{2}\left(\Gamma_{\ominus}\right), \quad \psi \in L_{2}\left(\Gamma_{\oplus}\right), \quad \frac{\partial \psi}{\partial x_{3}} \in L_{2}(\Omega) .
$$

(ii) Assume $\rho$ to be strictly positive. Then (25) can be modified to the estimate that does not involve the constant $C_{F, \Omega}$ :

$$
\left\|u-\hat{u}^{(1)}\right\| \mid \leqslant \sqrt{M_{1}^{2}+\tilde{M}_{2}^{2}}+M_{3},
$$

where $M_{1}$ and $M_{3}$ are from (26) and (27), respectively, and the functional $\tilde{M}_{2}^{2}$ is defined by

$$
\begin{gathered}
\tilde{M}_{2}^{2}:=\| \rho^{-1}\left(\left(f-\hat{f}_{0}\right)-\left(\rho^{2}-\tilde{\rho}_{0}^{2}\right) \hat{u}_{1, h}^{(1)}-\left(x_{3} \rho^{2}-\tilde{\rho}_{1}^{2}-\frac{1}{d(\hat{x})} \int_{d_{\ominus}}^{d_{\oplus}} a_{33}\right) \hat{u}_{2, h}^{(1)}\right. \\
\left.-\frac{\nabla d(\hat{x})}{d(\hat{x})} \cdot\left(\tilde{\mathbf{A}}_{p, 0} \hat{\nabla} \hat{u}_{1, h}^{(1)}+\tilde{\mathbf{A}}_{p, 1} \hat{\nabla} \hat{u}_{2, h}^{(1)}\right)+\frac{\partial \psi}{\partial x_{3}}\right) \|_{L_{2}(\Omega)}^{2} .
\end{gathered}
$$

(iii) If the function $\kappa$ is also strictly positive, then we obtain an upper bound that does not contain the constants $C_{F, \Omega}$ and $C_{\Gamma, R}$ :

$$
\left\|u-\hat{u}^{(1)}\right\| \leqslant \sqrt{M_{1}^{2}+\tilde{M}_{2}^{2}+\tilde{M}_{3 R}^{2}}+M_{3 \ominus \oplus,}
$$

where $M_{1}$ and $\tilde{M}_{2}^{2}$ are from (26) and (28), respectively, and

$$
\begin{gathered}
\tilde{M}_{3 R}^{2}:=\left\|\kappa^{-1 / 2}\left(\kappa\left(\hat{u}_{1, h}^{(1)}+x_{3} \hat{u}_{2, h}^{(1)}\right)+\left(\tilde{\mathbf{A}}_{p, 0} \hat{\nabla} \hat{u}_{1, h}^{(1)}+\tilde{\mathbf{A}}_{p, 1} \hat{\nabla} \hat{u}_{2, h}^{(1)}, 0\right) \cdot \boldsymbol{\nu}_{R}\right)\right\|_{L_{2}\left(\Gamma_{R}\right)}^{2}, \\
M_{3 \oplus \ominus}:=c_{1}^{-1}\left(C_{\Gamma, \ominus}\left\|F_{\ominus}-\left(\tilde{\mathbf{A}}_{p, 0} \hat{\nabla} \hat{u}_{1, h}^{(1)}+\tilde{\mathbf{A}}_{p, 1} \hat{\nabla} \hat{u}_{2, h}^{(1)}, 0\right) \cdot \boldsymbol{\nu}_{\ominus}-\psi \nu_{\ominus 3}\right\|_{L_{2}\left(\Gamma_{\ominus}\right)}\right. \\
\left.\quad+C_{\Gamma, \oplus}\left\|F_{\oplus}-\left(\tilde{\mathbf{A}}_{p, 0} \hat{\nabla} \hat{u}_{1, h}^{(1)}+\tilde{\mathbf{A}}_{p, 1} \hat{\nabla} \hat{u}_{2, h}^{(1)}, 0\right) \cdot \boldsymbol{\nu}_{\oplus}-\psi \nu_{\oplus 3}\right\|_{L_{2}\left(\Gamma_{\oplus}\right)}\right) .
\end{gathered}
$$

Remark 3.6. Recommendations for an appropriate choice of the auxiliary functions $\hat{\mathbf{y}}$ and $\psi$ can be found, e.g., in $[9,13]$.

\section{Numerical Tests}

\subsection{Test 1}

To analyze the performance of the proposed combined error majorant, we firstly consider a very simple two-dimensional problem

$$
\begin{aligned}
& -\Delta u+u=f \quad \text { in } \Omega \text {, } \\
& \nabla u \cdot \boldsymbol{\nu}_{\ominus}=F_{\ominus} \quad \text { at } x_{2}=d_{\ominus} \text {, }
\end{aligned}
$$




\begin{tabular}{ccccc}
\hline$d_{0}^{-1}$ & $\left\|u-\hat{u}_{h}^{(0)}\right\|$ & $E_{\text {disc }}^{h}$ & $E_{\text {mod }}^{(0), h}(\mathbf{y})$ & $i_{\text {eff }}(\mathbf{y})$ \\
\hline $10^{0}$ & 0.6195 & $2.4 \mathrm{e}-14$ & 3.48471 & 5.6238 \\
$10^{1}$ & 0.1299 & $2.8 \mathrm{e}-14$ & 0.29845 & 2.2961 \\
$10^{2}$ & 0.0408 & $2.0 \mathrm{e}-14$ & 0.05897 & 1.4445 \\
$10^{3}$ & 0.0129 & $2.2 \mathrm{e}-14$ & 0.01552 & 1.2024 \\
$10^{4}$ & 0.0041 & $2.3 \mathrm{e}-14$ & 0.00460 & 1.1268 \\
$10^{5}$ & 0.0013 & $2.3 \mathrm{e}-14$ & 0.00142 & 1.1029 \\
\hline
\end{tabular}

Table 1. Convergence of the efficiency index of the total error majorant depending on the domain thickness $d_{0}$ for $h=\frac{1}{32}$.

$$
\begin{aligned}
\nabla u \cdot \boldsymbol{\nu}_{\oplus} & =F_{\oplus} & & \text { at } x_{2}=d_{\oplus}, \\
u & =0 & & \text { at } x_{1}=0 \text { and } x_{1}=1,
\end{aligned}
$$

in the "sine shaped" domain

$$
\Omega:=\left\{\left(x_{1}, x_{2}\right) \in \mathbb{R}^{2} \mid x_{1} \in \hat{\Omega}:=(0,1), d_{\ominus}\left(x_{1}\right)<x_{2}<d_{\ominus}\left(x_{1}\right)\right\}
$$

with the upper and lower faces defined, respectively, by

$$
d_{\oplus}\left(x_{1}\right)=\sin \left(\pi x_{1}\right)+\frac{d_{0}}{2} \quad \text { and } \quad d_{\ominus}\left(x_{1}\right)=\sin \left(\pi x_{1}\right)-\frac{d_{0}}{2} .
$$

For this test problem, we compute the right-hand side function $f$ and the Neumann boundary condition functions $F_{\ominus}$ and $F_{\oplus}$ using the exact solution

$$
u\left(x_{1}, x_{2}\right)=\frac{x_{2}^{2}}{d_{0}} \sin \left(\pi x_{1}\right) .
$$

We note that since our test problem is a two-dimensional one, the corresponding reduced problem will be one-dimensional. In this case $\hat{x}=x_{1}$. We apply the zero-order model and solve it using MATLAB functions bvpinit and bvp4c, therefore, the numerical solution of the reduced problem is expected to be very accurately. We estimate the total error using the presented combined error majorant (10)-(18). By the calculation of the error majorant, we set for the auxiliary function $\mathbf{y}$ the averaged numerical solution of the flux and use the numerical differentiation to obtain div $\mathbf{y}$. Convergence of the exact error in the combined norm $\left\|u-\hat{u}_{h}^{(0)}\right\|$, the error majorant and the corresponding efficiency index

$$
i_{\mathrm{eff}}:=\frac{E_{\mathrm{mod}}^{(0), h}+E_{\mathrm{disc}}^{h}}{\left\|u-\hat{u}_{h}^{(0)}\right\| \|}
$$

as $d_{0} \rightarrow 0$ are presented in Table 1 .

The discretization error majorant $E_{\text {disc }}^{h}$ is neglected small, hence the total error estimate is almost completely explained by the modeling error. Although, the efficiency index is quite acceptable, it remains stable and does not converge to one. To improve the situation, we use the modeling error majorant (23), follow the recommendation from [9] and choose $\psi$ such that the last two terms of $M_{3}$ in (22) (i.e., the residual on the Neumann boundary conditions) are identically zero. We note that

$$
\begin{aligned}
\| F_{\ominus} & -\left(\tilde{\mathbf{A}}_{p, 0} \nabla \hat{u}_{h}^{(0)}, 0\right) \cdot \boldsymbol{\nu}_{\ominus}-\psi \nu_{\ominus 2} \|_{x_{2}=d_{\ominus}}^{2} \\
& =\int_{\hat{\Omega}}\left(\hat{F}_{\ominus}(\hat{x})-\left(\tilde{\mathbf{A}}_{p, 0} \hat{\nabla} \hat{u}_{h}^{(0)}, 0\right) \cdot \boldsymbol{\nu}_{\ominus}-\psi\left(\hat{x}, d_{\ominus}(\hat{x})\right) \nu_{\ominus 2}\right)^{2} \sqrt{1+\left|\nabla d_{\ominus}\right|^{2}} d \hat{x}
\end{aligned}
$$




\begin{tabular}{ccccc}
\hline$d_{0}^{-1}$ & $\left\|u-\hat{u}_{h}^{(0)}\right\|$ & $E_{\text {disc }}^{h}$ & $E_{\text {mod }}^{(0), h}\left(\psi_{1}\right)$ & $i_{\text {eff }}\left(\psi_{1}\right)$ \\
\hline $10^{0}$ & 0.6195 & $2.4 \mathrm{e}-14$ & 0.8970 & 1.4479 \\
$10^{1}$ & 0.1299 & $2.8 \mathrm{e}-14$ & 0.1422 & 1.0949 \\
$10^{2}$ & 0.0408 & $2.0 \mathrm{e}-14$ & 0.0412 & 1.0099 \\
$10^{3}$ & 0.0129 & $2.2 \mathrm{e}-14$ & 0.0129 & 1.0010 \\
$10^{4}$ & 0.0041 & $2.3 \mathrm{e}-14$ & 0.0041 & 1.0001 \\
$10^{5}$ & 0.0013 & $2.3 \mathrm{e}-14$ & 0.0013 & 1.0000 \\
\hline
\end{tabular}

Table 2. Convergence of the efficiency index of the total error majorant depending on the domain thickness $d_{0}$ for $h=\frac{1}{32}$.

(analogously for the norm for $x_{2}=d_{\oplus}$ ) and set

$$
\psi(\hat{x})=\psi_{1}(\hat{x}):=\hat{\alpha}(\hat{x}) x_{2}+\hat{\beta}(\hat{x}),
$$

where the functions $\hat{\alpha}$ and $\hat{\beta}\left(\hat{\alpha}, \hat{\beta} \in L_{2}(\hat{\Omega})\right)$ are uniquely defined by the condition

$$
\begin{array}{ll}
\psi \nu_{\ominus 2}=\hat{F}_{\ominus}-\left(\tilde{\mathbf{A}}_{p, 0} \hat{\nabla} \hat{u}_{h}^{(0)}, 0\right) \cdot \boldsymbol{\nu}_{\ominus} & \text { at } x_{2}=d_{\ominus}, \\
\psi \nu_{\oplus 2}=\hat{F}_{\oplus}-\left(\tilde{\mathbf{A}}_{p, 0} \hat{\nabla} \hat{u}_{h}^{(0)}, 0\right) \cdot \boldsymbol{\nu}_{\oplus} & \text { at } x_{2}=d_{\oplus} .
\end{array}
$$

The corresponding $\hat{\alpha}$ and $\hat{\beta}$ have the form

$$
\hat{\alpha}=\frac{1}{d}\left(\frac{\hat{G}_{\oplus}}{\nu_{\oplus 2}}-\frac{\hat{G}_{\ominus}}{\nu_{\ominus 2}}\right) \quad \text { and } \quad \hat{\beta}=\frac{1}{d}\left(\frac{\hat{G}_{\ominus}}{\nu_{\ominus 2}} d_{\oplus}-\frac{\hat{G}_{\oplus}}{\nu_{\oplus 2}} d_{\ominus}\right)
$$

with

$$
\hat{G}_{\ominus}:=\hat{F}_{\ominus}-\left(\tilde{\mathbf{A}}_{p, 0} \hat{\nabla} \hat{u}_{h}^{(0)}, 0\right) \cdot \boldsymbol{\nu}_{\ominus} \quad \text { and } \quad \hat{G}_{\oplus}:=\hat{F}_{\oplus}-\left(\tilde{\mathbf{A}}_{p, 0} \hat{\nabla} \hat{u}_{h}^{(0)}, 0\right) \cdot \boldsymbol{\nu}_{\oplus} \cdot
$$

It is easy to see that $\hat{\alpha}, \hat{\beta} \in L_{2}(\hat{\Omega})$. Hence, $\psi$ from (29) and its derivative in $x_{2}$-direction belong to $L_{2}(\Omega)$.

Now, we obtain for the right-hand side terms of (23) the following representation:

$$
\begin{aligned}
M_{1}= & \left(\int_{\hat{\Omega}} d(\hat{x})\left(\tilde{\mathbf{B}}_{p, 0} \tilde{\mathbf{A}}_{p, 0}-\mathbf{I}\right) \hat{\nabla} \hat{u}_{h}^{(0)} \cdot \tilde{\mathbf{A}}_{p, 0} \hat{\nabla} \hat{u}_{h}^{(0)} d \hat{x}\right. \\
& \left.\quad+\int_{\Omega}\left(b_{22}\left(\hat{\alpha}(\hat{x}) x_{2}+\hat{\beta}(\hat{x})\right)^{2}+2\left(\mathbf{b}_{2} \cdot\left(\tilde{\mathbf{A}}_{p, 0} \hat{\nabla} \hat{u}_{h}^{(0)}, 0\right)\right)\left(\hat{\alpha}(\hat{x}) x_{2}+\hat{\beta}(\hat{x})\right)\right) d x\right)^{1 / 2}, \\
\tilde{M}_{2}= & \left\|\rho^{-1}\left(\left(f-\tilde{f}_{0}\right)-\left(\rho^{2}-\tilde{\rho}_{0}^{2}\right) \hat{u}_{h}^{(0)}\right)\right\|_{L_{2}(\Omega)}, \\
M_{3}= & c_{1}^{-1}\left(C_{\Gamma, R}\left\|\kappa \hat{u}_{h}^{(0)}+\left(\tilde{\mathbf{A}}_{p, 0} \hat{\nabla} \hat{u}_{h}^{(0)}, 0\right) \cdot \boldsymbol{\nu}_{R}\right\|_{L_{2}\left(\Gamma_{R}\right)}\right) .
\end{aligned}
$$

In our test problem, we do not have the Robin boundary condition and in addition

$$
d_{\oplus}=d_{\ominus}+d_{0}
$$

Hence, we obtain $M_{\text {mod }}\left(\psi_{1}\right)=M_{1}\left(\psi_{1}\right)+\tilde{M}_{2}\left(\psi_{1}\right)$ with

$$
\begin{aligned}
M_{1}\left(\psi_{1}\right) & =\left(\int_{\Omega} \psi_{1}^{2} d x\right)^{1 / 2}=\left(\int_{\hat{\Omega}} \int_{d_{\ominus}}^{d_{\ominus}+d_{0}}\left(\hat{\alpha}^{2} x_{2}^{2}+2 \hat{\alpha} \hat{\beta} x_{2}+\hat{\beta}^{2}\right) d x_{2} d \hat{x}\right)^{1 / 2} \\
& =\left(d_{0} \int_{\hat{\Omega}}\left(\hat{\alpha}^{2} \frac{d_{\oplus}^{2}+d_{\oplus} d_{\ominus}+d_{\ominus}^{2}}{3}+\hat{\alpha} \hat{\beta}\left(d_{\oplus}+d_{\ominus}\right)+\hat{\beta}^{2}\right) d \hat{x}\right)^{1 / 2} \\
\tilde{M}_{2}\left(\psi_{1}\right) & =\left\|\rho^{-1}\left(f-\tilde{f}_{0}-\left(\rho^{2}-\tilde{\rho}_{0}^{2}\right) \hat{u}_{h}^{(0)}\right)\right\|_{L_{2}(\Omega)} .
\end{aligned}
$$




\begin{tabular}{cccccc}
\hline$d_{0}^{-1}$ & $\left\|u-\hat{u}_{h}^{(0)}\right\|$ & $E_{\text {disc }}^{h}$ & $M_{1}\left(\psi_{1}\right)$ & $\tilde{M}_{2}\left(\psi_{1}\right)$ & $i_{\text {eff }}\left(\psi_{1}\right)$ \\
\hline $10^{0}$ & 0.2615 & $3.4 \mathrm{e}-15$ & 0.10201 & 0.28765 & 1.4903 \\
$10^{1}$ & 0.0751 & $2.8 \mathrm{e}-14$ & 0.00323 & 1.22951 & 16.418 \\
$10^{2}$ & 0.0237 & $2.0 \mathrm{e}-13$ & 0.00010 & 3.89840 & 164.37 \\
$10^{3}$ & 0.0075 & $2.2 \mathrm{e}-12$ & $3.23 \mathrm{e}-6$ & 12.3281 & 1643.7 \\
$10^{4}$ & 0.0024 & $2.3 \mathrm{e}-11$ & $1.25 \mathrm{e}-7$ & 39.4675 & 16444 \\
$10^{5}$ & 0.0008 & $4.3 \mathrm{e}-14$ & $4.00 \mathrm{e}-9$ & 131.656 & 164570 \\
\hline
\end{tabular}

Table 3. Convergence of the efficiency index of the total error majorant depending on the domain thickness $d_{0}$ for $h=\frac{1}{32}$.

In such a case, see Table 2 , the majorant $E_{\text {mod }}^{(0), h}\left(\psi_{1}\right)=M_{\bmod }\left(\psi_{1}\right)+E_{\text {disc }}^{h}$ demonstrates the asymptotic exactness, and, moreover, the index of efficiency behaves like $1+\mathcal{O}\left(d_{0}\right)$.

\subsection{Test 2}

In the second test, we consider the same two-dimensional problem with the exact solution

$$
u\left(x_{1}, x_{2}\right)=\frac{x_{2}^{3}}{d_{0}^{2}} \sin \left(\pi x_{2}\right),
$$

use the zero-order model as the reduced formulation and solve this reduced problem with MATLAB to obtain an accurate numerical solution. For the modeling error, we use the representation (23) and set again

$$
\psi(\hat{x})=\psi_{1}(\hat{x}):=\hat{\alpha}(\hat{x}) x_{2}+\hat{\beta}(\hat{x}) .
$$

Here, $\tilde{M}_{2}$ becomes dominating and the whole error estimate grows unboundedly, as can be seen in Table 3.

It is rather clear that the problem originates from the bad choice of the auxiliary function $\psi$ that is supposed to approximate $\frac{\partial u}{\partial x_{2}}$. For the chosen function $u\left(x_{1}, x_{2}\right)=\frac{x_{2}^{3}}{d_{0}^{2}} \sin \left(\pi x_{1}\right)$, the derivative is quadratic and cannot be adequately represented by the linear function $\psi_{1}$. We cure the situation setting

$$
\psi=\psi_{2}:=\psi_{1}+\hat{\eta}\left(x_{1}\right)\left(x_{2}^{2}-\frac{d_{0}^{2}}{4}\right)
$$

where $\hat{\eta}$ is an arbitrary function from $L_{2}(\hat{\Omega})$. We obtain the improved majorant

$$
E_{\text {mod }}^{(0), h}\left(\psi_{2}\right)=M_{\text {mod }}\left(\psi_{2}\right)+E_{\text {disc }}^{h}=M_{1}\left(\psi_{2}\right)+\tilde{M}_{2}\left(\psi_{2}\right)+E_{\text {disc }}^{h}
$$

with

$$
\begin{aligned}
& M_{1}\left(\psi_{2}\right)=\left\|\psi_{1}+\hat{\eta}\left(x_{1}\right)\left(x_{2}^{2}-\frac{d_{0}^{2}}{4}\right)\right\|_{L_{2}(\Omega)}, \\
& \tilde{M}_{2}\left(\psi_{2}\right)=\left\|\rho^{-1}\left((f-\hat{f})-\left(\rho^{2}-\tilde{\rho}_{0}^{2}\right) \hat{u}_{h}^{(0)}+2 \hat{\eta}\left(x_{1}\right) x_{2}\right)\right\|_{L_{2}(\Omega)} .
\end{aligned}
$$

The functional $M_{\bmod }\left(\psi_{2}\right)$ can be minimized over the space of piecewise-constant functions. The properties of the error majorant $E_{\bmod }\left(\psi_{2}\right)^{(0), h}$ can be observed in Table 4: The majorant decays with the optimal rate as $d_{0}$ tends to zero, it is a reliable upper bound for the exact error in the combined norm and demonstrates the asymptotic exactness with the efficiency index behaving like $1+\mathcal{O}\left(d_{0}\right)$. 


\begin{tabular}{ccccc}
\hline$d_{0}^{-1}$ & $\left\|u-\hat{u}_{h}^{(0)}\right\|$ & $E_{\text {disc }}^{h}$ & $E_{\text {mod }}^{(0), h}\left(\psi_{2}\right)$ & $i_{\text {eff }}\left(\psi_{2}\right)$ \\
\hline $10^{0}$ & 0.2615 & $3.4 \mathrm{e}-15$ & 0.3727 & 1.4252 \\
$10^{1}$ & 0.0751 & $2.8 \mathrm{e}-14$ & 0.0129 & 1.1712 \\
$10^{2}$ & 0.0237 & $2.0 \mathrm{e}-13$ & 0.0241 & 1.0162 \\
$10^{3}$ & 0.0075 & $2.2 \mathrm{e}-12$ & 0.0075 & 1.0017 \\
$10^{4}$ & 0.0024 & $2.3 \mathrm{e}-11$ & 0.0024 & 1.0002 \\
$10^{5}$ & 0.0008 & $4.3 \mathrm{e}-14$ & 0.0008 & 1.0000 \\
\hline
\end{tabular}

Table 4. Convergence of the efficiency index of the total error majorant depending on the domain thickness $d_{0}$ for $h=\frac{1}{32}$.

\subsection{Test 3}

In the following test, we consider a three-dimensional diffusion-reaction problem

$$
\begin{aligned}
-\Delta u+2 u & =f & & \text { in } \Omega, \\
\nabla u \cdot \boldsymbol{\nu}_{\ominus} & =F_{\ominus} & & \text { on } \partial \Omega_{\ominus}, \\
\nabla u \cdot \boldsymbol{\nu}_{\oplus} & =F_{\oplus} & & \text { on } \partial \Omega_{\oplus}, \\
u & =0 & & \text { on } \partial \Omega_{D},
\end{aligned}
$$

where the domain $\Omega$ is defined by

$$
\Omega:=\left\{\left(x_{1}, x_{2}, x_{3}\right) \in \mathbb{R}^{3} \mid\left(x_{1}, x_{2}\right) \in \hat{\Omega}:=(-1,1)^{2},-\frac{d_{0}}{2}<x_{3}<\frac{d_{0}}{2}\right\}
$$

with the boundaries

$$
\begin{aligned}
& \partial \Omega_{\ominus}:=\left\{\left(x_{1}, x_{2}, x_{3}\right) \in \mathbb{R}^{3} \mid\left(x_{1}, x_{2}\right) \in \hat{\Omega}:=(-1,1)^{2}, x_{3}=-\frac{d_{0}}{2}\right\}, \\
& \partial \Omega_{\oplus}:=\left\{\left(x_{1}, x_{2}, x_{3}\right) \in \mathbb{R}^{3} \mid\left(x_{1}, x_{2}\right) \in \hat{\Omega}:=(-1,1)^{2}, x_{3}=\frac{d_{0}}{2}\right\},
\end{aligned}
$$

and

$$
\partial \Omega_{D}:=\partial \Omega \backslash\left(\partial \Omega_{\ominus} \cup \partial \Omega_{\oplus}\right) .
$$

We compute the right-hand sides of the equation and the boundary condition using the exact solution

$$
u\left(x_{1}, x_{2}\right)=\frac{x_{3}^{2}}{d_{0}} \sin \left(\pi x_{1}\right) \sin \left(\pi x_{2}\right) .
$$

To solve the problem approximately, we assume the zero-order model. For the modeling error estimate in our domain with plane parallel faces, we use the approach

$$
\psi(\hat{x})=\psi_{1}(\hat{x})=\alpha(\hat{x}) x_{3}+\beta(\hat{x})
$$

and obtain for the auxiliary function $\psi$ the representation

$$
\psi=\psi_{1}=\frac{\hat{F}_{\oplus}+\hat{F}_{\ominus}}{d_{0}} x_{3}+\frac{\hat{F}_{\oplus}-\hat{F}_{\ominus}}{d_{0}} .
$$

In the case of the zero-order model, the term $M_{1}$ reduces to

$$
M_{1}=\left(\frac{d_{0}}{3} \int_{\hat{\Omega}}\left(\hat{F}_{\oplus}^{2}-\hat{F}_{\oplus} \hat{F}_{\ominus}+\hat{F}_{\ominus}^{2}\right)\right)^{1 / 2},
$$




\begin{tabular}{ccccc}
\hline$d_{0}^{-1}$ & $\left\|u-\hat{u}_{h}^{(0)}\right\|$ & $E_{\text {disc }}^{h}$ & $E_{\text {mod }}^{(0), h}\left(\psi_{1}\right)$ & $i_{\text {eff }}\left(\psi_{1}\right)$ \\
\hline $10^{0}$ & $7.45 \mathrm{e}-01$ & $7.39 \mathrm{e}-02$ & $9.65 \mathrm{e}-01$ & 1.3944 \\
$10^{1}$ & $1.83 \mathrm{e}-01$ & $7.39 \mathrm{e}-03$ & $2.00 \mathrm{e}-01$ & 1.1315 \\
$10^{2}$ & $5.77 \mathrm{e}-02$ & $7.39 \mathrm{e}-04$ & $5.88 \mathrm{e}-02$ & 1.0310 \\
$10^{3}$ & $1.83 \mathrm{e}-02$ & $7.39 \mathrm{e}-05$ & $1.83 \mathrm{e}-02$ & 1.0086 \\
$10^{4}$ & $5.77 \mathrm{e}-03$ & $7.39 \mathrm{e}-06$ & $5.78 \mathrm{e}-03$ & 1.0026 \\
$10^{5}$ & $1.83 \mathrm{e}-03$ & $7.39 \mathrm{e}-07$ & $1.83 \mathrm{e}-03$ & 1.0008 \\
\hline
\end{tabular}

Table 5. Convergence of the efficiency index of the total error majorant depending on the domain thickness $d_{0}$ for $h=\frac{1}{32}$ in the case of the zero-order model.

as well as

$$
M_{2}=\left\|\rho^{-1}\left(f-\tilde{f}-\left(\rho-\tilde{\rho}_{0}^{2}\right) \hat{u}_{h}^{(0)}\right)\right\|_{L_{2}(\Omega)} .
$$

For the discretization error majorant, we make the simplest choice of the auxiliary function $\mathbf{y}$ setting it equal to the averaged numerical flux and using the numerical differentiation to obtain $\operatorname{div} \mathbf{y}$. Now, we set the amount of knots $n=961$, i.e., $h=\frac{1}{32}$, and solve the reduced problem with MATLAB using functions assempde and pdecgrad. We observe the convergence of the exact error in the combined norm and of the error majorant as $d_{0}$ tends to zero.

The corresponding numerical results demonstrate the high efficiency of the proposed combined error majorant, see Table 5, Figure 2 and Figure 3. Both the exact error and the combined majorant vanish with the optimal rate $\mathcal{O}\left(d_{0}^{1 / 2}\right)$.

\subsection{Test 4}

In order to illustrate the usage of the presented error majorant by the solution of the partial differential equations, we consider the test problem (30)-(34) that has to be solved in the domain

$$
\Omega:=\left\{\left(x_{1}, x_{2}, x_{3}\right) \in \mathbb{R}^{3} \mid\left(x_{1}, x_{2}\right) \in \hat{\Omega}:=(-1,1)^{2},-0.01<x_{3}<0.01\right\}
$$

with the accuracy

$$
\frac{\left\|u-u_{h}^{(m)}\right\|}{\left\|u_{h}^{(m)}\right\|} \leqslant 0.1,
$$

where $u_{h}^{(m)}$ is the numerical solution of the reduced $m$-order model. With the combined error majorant, it holds

$$
\left\|u-u_{h}^{(m)}\right\| \| \leqslant E_{\text {mod }}^{(m), h}+E_{\text {disc }}^{h} .
$$

Hence, we can use the reference quantity

$$
\frac{E_{\bmod }^{(m), h}\left(\psi_{1}\right)+E_{\mathrm{disc}}^{h}}{\left\|\hat{u}_{h}^{(m)}\right\| \|}
$$

to verify the desired accuracy. At first, we apply a reduced model and solve it on some coarse mesh. Then, we calculate the modeling and discretization error estimates $E_{\text {mod }}^{(m),}$ and $E_{\text {disc }}^{h}$. Then, we obtain the proposed combined error estimate of the total error. If the discretization error estimate becomes dominating then it is worth solving the reduced model 


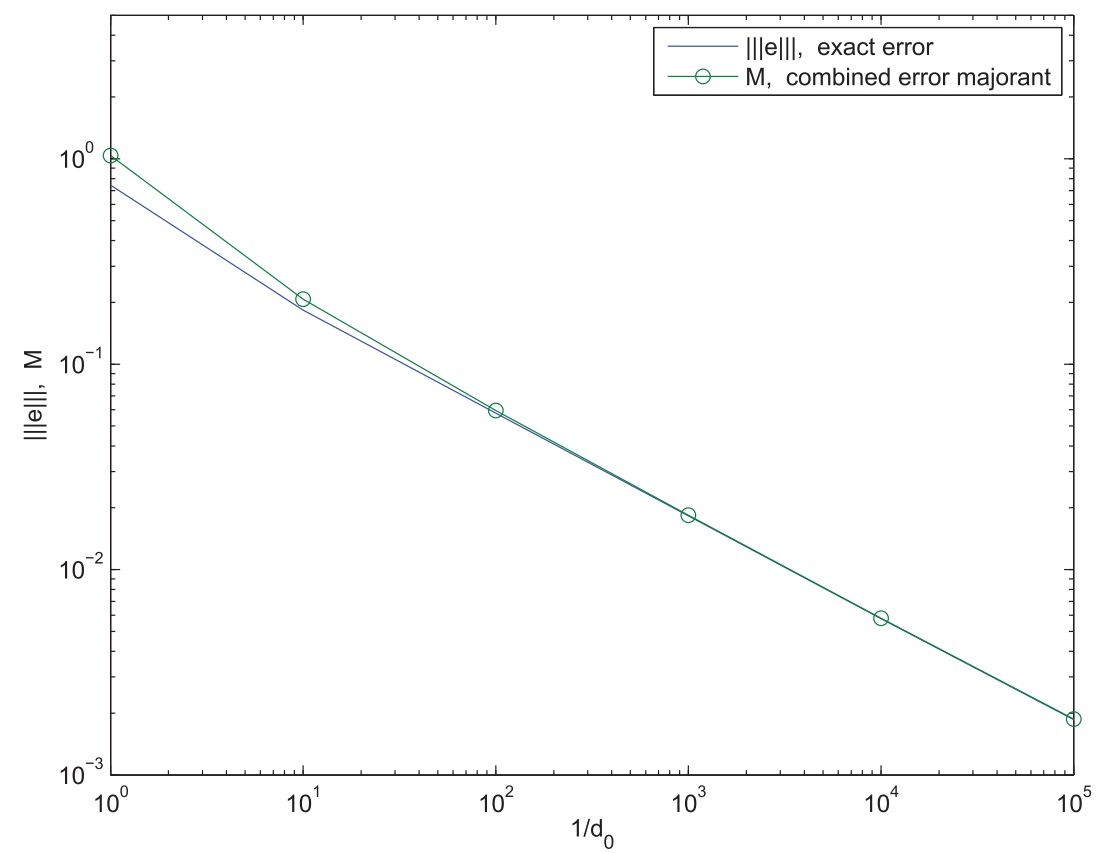

Figure 2. Plot of the exact error and the combined error majorant in the case of $h=\frac{1}{32}$ in the case of the zero-order model.

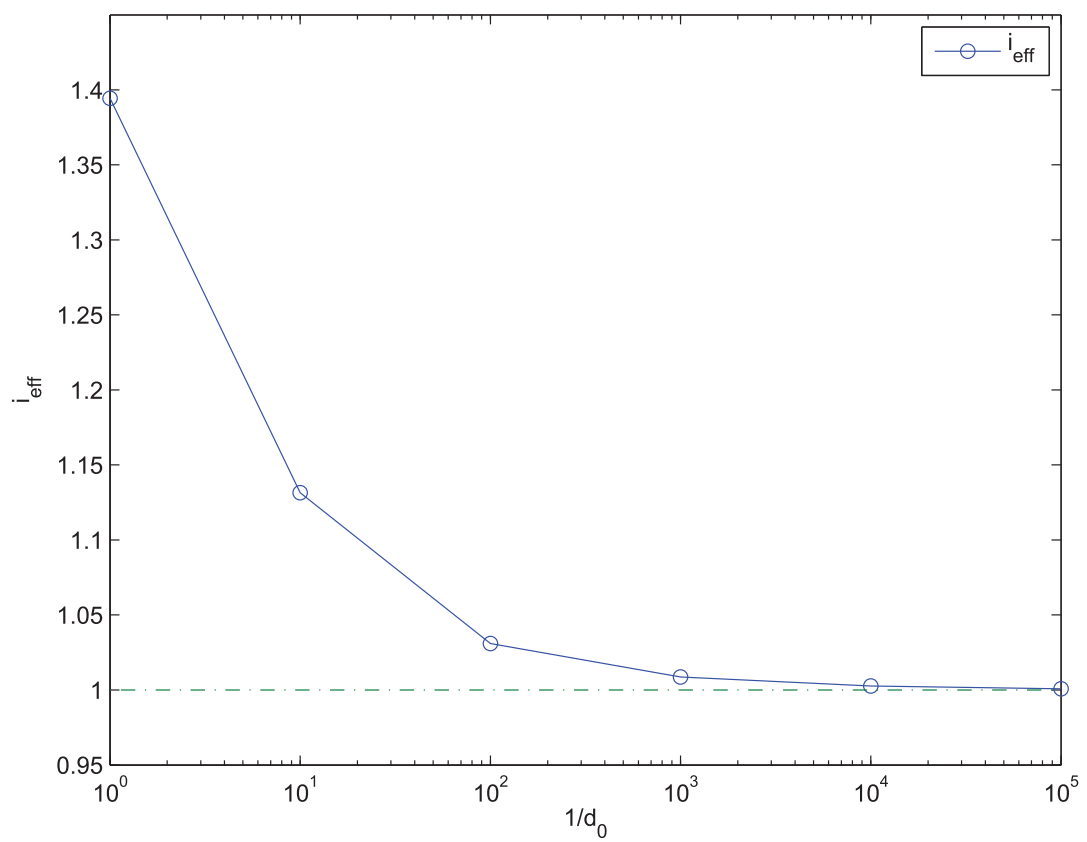

Figure 3. Plot of the efficiency index in the case of $h=\frac{1}{32}$ in the case of the zero-order model. 


\begin{tabular}{ccccc}
\hline$d_{0}^{-1}$ & $n$ & $E_{\text {mod }}^{(0), h}\left(\psi_{1}\right)$ & $E_{\text {disc }}^{h}$ & $\frac{E_{\text {mod }}^{(0), h}\left(\psi_{1}\right)+E_{\text {disc }}^{h}}{\left\|\hat{u}_{h}^{(0)}\right\|}$ \\
\hline $10^{2}$ & 961 & $5.879 \mathrm{e}-03$ & $7.390 \mathrm{e}-03$ & 8.386 \\
\hline
\end{tabular}

Table 6. Reference relation in the case of Model 1 for $h=\frac{1}{32}$.

\begin{tabular}{ccccc}
\hline$d_{0}^{-1}$ & $n$ & $E_{\text {mod }}^{(1), h}\left(\psi_{1}\right)$ & $E_{\text {disc }}^{h}$ & $\frac{E_{\text {mod }}^{(1), h}\left(\psi_{1}\right)+E_{\text {disc }}^{h}}{\left\|\hat{u}_{h}^{(1)}\right\|}$ \\
\hline $10^{2}$ & 961 & $5.308 \mathrm{e}-03$ & $4.727 \mathrm{e}-03$ & 0.221 \\
\hline
\end{tabular}

Table 7. Reference relation in the case of Model 2 for $h=\frac{1}{32}$.

\begin{tabular}{ccccc}
\hline$d_{0}^{-1}$ & $n$ & $E_{\bmod }^{(2), h}\left(\psi_{1}\right)$ & $E_{\text {disc }}^{h}$ & $\frac{E_{\text {mod }}^{(2), h}\left(\psi_{1}\right)+E_{\mathrm{disc}}^{h}}{\left\|\hat{u}_{h}^{(2)}\right\|}$ \\
\hline $10^{2}$ & 961 & $1.443 \mathrm{e}-06$ & $4.681 \mathrm{e}-02$ & 0.124 \\
$10^{2}$ & 3969 & $1.443 \mathrm{e}-06$ & $3.381 \mathrm{e}-02$ & 0.091 \\
\hline
\end{tabular}

Table 8. Reference relation in the case of Model 4 for $h=\frac{1}{32}$ and $h=\frac{1}{64}$.

problem on the finer mesh in order to get a more exact solution. In the opposite case, the mesh refinement does not provide any exacter solution of the basic problem.

In our test, we use the zero-order model (e.g., Model 1), solve this reduced problem on the (coarse) mesh with $h=\frac{1}{32}$ and estimate the modeling and discretization errors $E_{\bmod }^{(0), h}$ and $E_{\text {disc }}^{h}$ as in Test 3. The corresponding numerical results are presented in Table 6.

For the reference relation, it holds

$$
\frac{E_{\text {mod }}^{(0), h}\left(\psi_{1}\right)+E_{\text {disc }}^{h}}{\left\|\hat{u}_{h}^{(0)}\right\| \|}>0.1 .
$$

Furthermore, the modeling error is dominating, hence the solution can be improved by using a higher-order model, e.g., Model 2. The corresponding numerical results with the improved reduced model are presented in Table 7 .

Hence, we have still

$$
\frac{E_{\mathrm{mod}}^{(1), h}\left(\psi_{1}\right)+E_{\mathrm{disc}}^{h}}{\left\|\hat{u}_{h}^{(1)}\right\|}>0.1
$$

and $E_{\text {mod }}^{(1), h}\left(\psi_{1}\right)>E_{\text {disc }}^{h}$, thus we should pass to the more accurate Model 4. Here (see Table 8), the reference relation

$$
\frac{E_{\bmod }^{(2), h}\left(\psi_{1}\right)+E_{\text {disc }}^{h}}{\left\|\hat{u}_{h}^{(2)}\right\| \|}=0.124>0.1
$$

and $E_{\text {mod }}^{(2), h}\left(\psi_{1}\right)<E_{\text {disc }}^{h}$, therefore we should refine the mesh.

For the mesh $h=\frac{1}{64}$ the desired accuracy is reached. As shown in Figure 4, the total error of the coarse Model 1 exceeds the total error of the more exact Model 2 and Model 4 for all the values of the thickness $d_{0}$. Comparing the reference relations

$$
\frac{E_{\text {mod }}^{(0), h}\left(\psi_{1}\right)+E_{\text {disc }}^{h}}{\left\|\hat{u}_{h}^{(0)}\right\| \|}, \quad \frac{E_{\text {mod }}^{(1), h}\left(\psi_{1}\right)+E_{\text {disc }}^{h}}{\left\|\hat{u}_{h}^{(1)}\right\| \|} \text { and } \frac{E_{\text {mod }}^{(2), h}\left(\psi_{1}\right)+E_{\text {disc }}^{h}}{\left\|\hat{u}_{h}^{(2)}\right\| \|},
$$




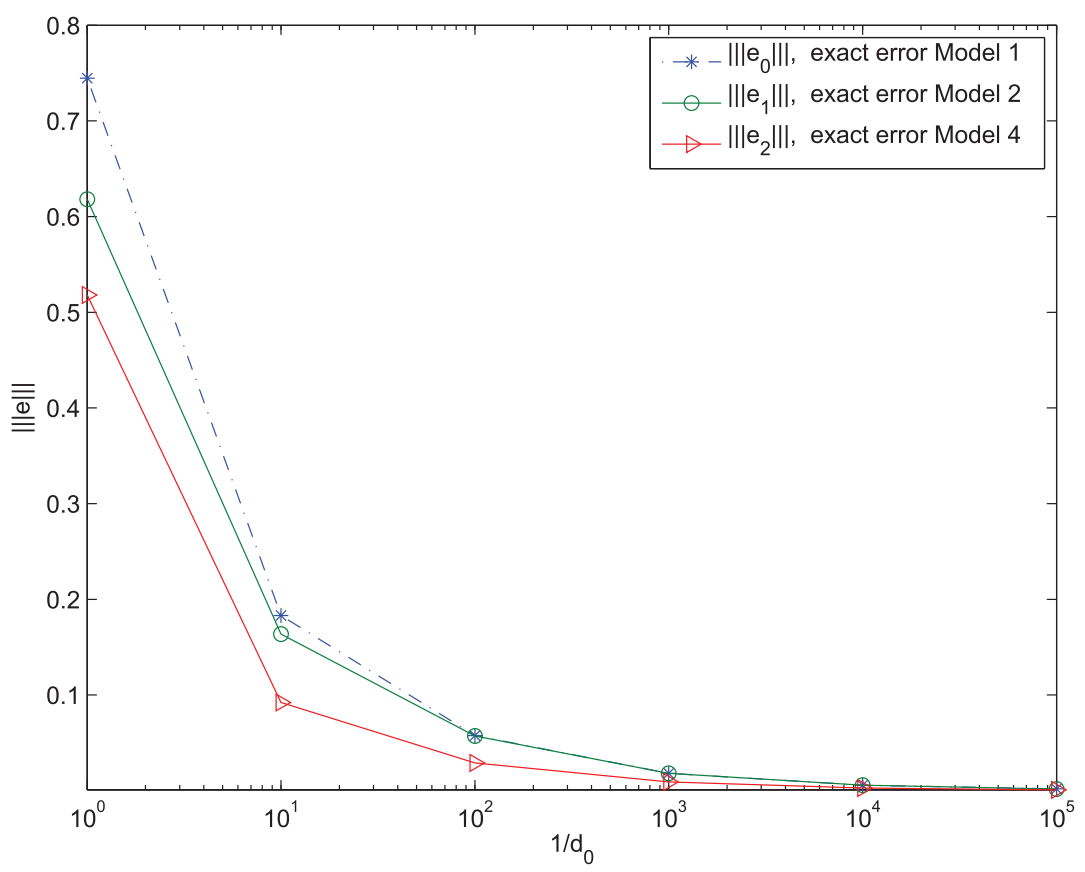

Figure 4. Plot of the exact total error for the zero-order (Model 1), the first-order (Model 2) and the second-order (Model 4) models in the case of $h=\frac{1}{32}$.

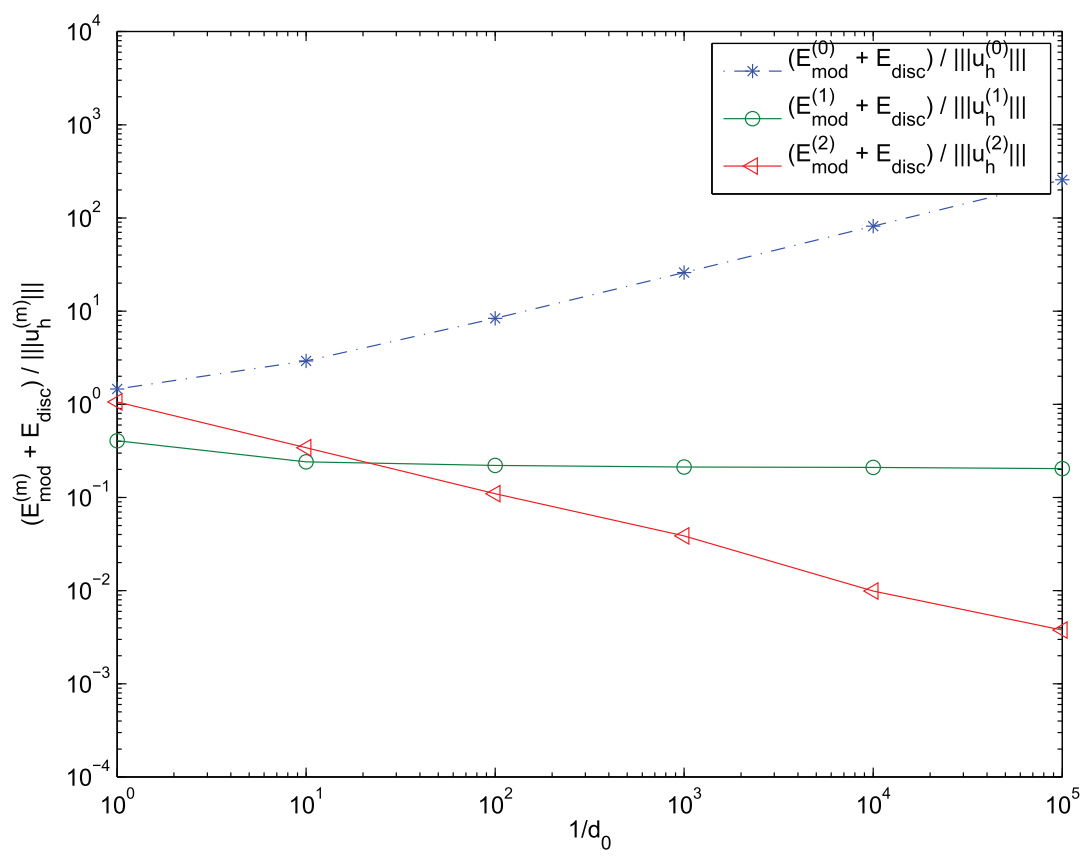

Figure 5. Plot of the reference relation $\frac{E_{\text {mod }}^{(m)}\left(\psi_{1}\right)+E_{\text {disc }}}{\left\|\hat{u}_{h}^{(m)}\right\|}$ for Model 1 and Model 4 in the case of $h=\frac{1}{32}$. 
we see in Figure 5 that the reference relation in the coarser model increases or does not vary significantly, and in the exacter model it decreases with the decreasing thickness of the domain. The obtained results meet our expectations of the theoretical part completely.

\section{References}

[1] A. L. Alessandrini, D. N. Arnold, R. S. Falk, and A. L. Madureira, Derivation and justification of plate models by variational methods, in: CRM Proceedings and Lecture Notes, 21, American Mathematical Society, Providence, 1996.

[2] I. Babuška and M. Vogelius, On a dimensional reduction method I. The optimal selection of basis functions, Math. Comp., 37 (1981), pp. 31-46.

[3] I. Babuška, I. Lee, and C. Schwab, A posteriori error estimation for hierarchic models of elliptic boundary value problems on thin domains, SIAM J. Numer. Anal., 33 (1996), pp. 221-246.

[4] P. G. Ciarlet, Plates and Junctions in Elastic Multi-Structures. An Asymptotic Analysis, Recherches en Mathematiques Appliquees, Springer, Berlin, 1990.

[5] P. G. Ciarlet and P. Destuynder, A justification of a nonlinear model in plate theory, Comput. Methods Appl. Mech. Engrg., 17/18 (1979), pp. 227-258.

[6] P. Neittaanmäki and S. Repin, Reliable Methods for Computer Simulation, Stud. Math. Appl., 33, Elsevier, Amsterdam, 2004.

[7] S. Repin, Functional approach to locally based a posteriori error estimates for elliptic and parabolic problems, in: Numerical Mathematics and Advanced Applications, Springer, Berlin, 2006, pp. 135-150.

[8] S. Repin, A Posteriori Estimates for Partial Differential Equations, Radon Ser. Comput. Appl. Math., 4, De Gruyter, Berlin, 2008.

[9] S. Repin and T. Samrowski, Estimates of dimension reduction errors for stationary reaction-diffusion problems, J. Math. Sci. (NY), 173 (2011), no. 6, pp. 803-821.

[10] S. Repin, T. Samrowski, and S. Sauter, Combined a posteriori modeling-discretization error estimate for elliptic problems with complicated interfacees, ESAIM: Math. Model. Numer. Anal., 46 (2012), no. 6, pp. 1389-1405.

[11] S. Repin and S. Sauter, Functional a posteriori estimates for the reaction-diffusion problem, C. R. Math. Acad. Sci. Paris, 343 (2006), no. 5, pp. 349-354.

[12] S. Repin and S. Sauter, Estimates of the modeling error for the Kirchhoff-Love plate model, C. R. Math. Acad. Sci. Paris, 348 (2010), no. 17-18, pp. 1039-1041.

[13] S. Repin, S. Sauter, and A. Smolianski, A posteriori estimation of dimension reduction errors for elliptic problems in thin domains, SIAM J. Numer. Anal., 42 (2004), pp. 1435-1451. 